\title{
miRNA-223 upregulated by MYOD inhibits myoblast proliferation by repressing IGF2 and facilitates myoblast differentiation by inhibiting ZEB1
}

\author{
Guihuan $\mathrm{Li}^{1,2,3,4}$, Wen Luo ${ }^{1,2,4}$, Bahareldin A Abdalla ${ }^{1,2}$, Hongjia Ouyang ${ }^{1,2}$, Jiao $\mathrm{Yu}^{1,2}$, Fan $\mathrm{Hu}^{1,2}$, Qinghua Nie ${ }^{\star, 1,2}$ and Xiquan Zhang ${ }^{1,2}$
}

Skeletal muscle differentiation can be regulated by various transcription factors and non-coding RNAs. In our previous work, miR-223 is differentially expressed in the skeletal muscle of chicken with different growth rates, but its role, expression and action mechanism in muscle development still remains unknown. Here, we found that MYOD transcription factor can upregulate miR-223 expression by binding to an E-box region of the gga-miR-223 gene promoter during avian myoblast differentiation. IGF2 and ZEB1 are two target genes of miR-223. The target inhibition of miR-223 on IGF2 and ZEB1 are dynamic from proliferation to differentiation of myoblast. miR-223 inhibits IGF2 expression only in the proliferating myoblast, whereas it inhibits ZEB1 mainly in the differentiating myoblast. The inhibition of IGF2 by miR-223 resulted in the repression of myoblast proliferation. During myoblast differentiation, miR-223 would be upregulated owing to the promoting effect of MYOD, and the upregulation of miR-223 would inhibit ZEB1 to promote myoblast differentiation. These results not only demonstrated that the well-known muscle determination factor MYOD can promote myoblast differentiation by upregulate miR-223 transcription, but also identified that miR-223 can influence myoblast proliferation and differentiation by a dynamic manner regulates the expression of its target genes.

Cell Death and Disease (2017) 8, e3094; doi:10.1038/cddis.2017.479; published online 5 October 2017

MicroRNAs (miRNAs) are small non-coding RNA of 20-25 nucleotides that mainly transcript from introns or intergenic regions, having critical roles in the regulation of gene expression at posttranscriptional levels. ${ }^{1}$ It have been reported that miRNAs widely participate in chicken embryo growth, maturation, muscle cell proliferation and differentiation, cell migration, dosage compensation effect of $Z$ chromosome and a series of life activities. ${ }^{2-7}$ In our preliminary study, we have used high-throughput RNA sequencing to study breast muscle transcriptome in Recessive White Rock (fast-growing chicken) and Xinghua chicken (slow-growing chicken), and pointed out that miR-223 can be used as a candidate gene associating with broiler growth. ${ }^{8}$

The miR-223 is highly conserved among vertebrates. As the first miRNA identified in human hemopoietic system, ${ }^{9}$ miR-223 was confirmed to participate in the regulation of hematopoietic cell proliferation and differentiation, ${ }^{10}$ and become a research hotspot. There are some competitive binding sites for NFI-A and C/EBP alpha in the upstream of $m i R-223$. C/EBP alpha enhances miR-223 transcription activity, whereas NFI-A inhibits the transcription activity of $m i R-223 .{ }^{10}$ The upregulation of miR-223 expression will promote granulocyte and megakaryocyte generation, and the decline of miR-223 expression will cause hematopoietic stem cells to differentiate into erythrocyte. ${ }^{11,12}$ In addition, miR-223 was confirmed to regulate cell proliferation, differentiation, migration and signal transduction in mammals. ${ }^{13}$ However, the roles of miR-223 in muscle development still remain unclear.

Insulin-like growth factor-2 (IGF2) is a member of insulin-like growth factor family that can play a role in all insulin-activated target cells. ${ }^{14}$ It is well-known that IGF2 can be acted as the intermediate messenger of growth hormone, which can transfer the growth hormone to target organs, and regulate the development of organisms. ${ }^{15}$ IGF2 is conserved during evolution, the gene structure and exons location in chicken are similar to that in mammal, although the non-coding sequences were very different. ${ }^{16}$ In mammals, IGF2 is a kind of multifunctional cell regulation factor that has an important role in the muscle cell proliferation and differentiation. ${ }^{17}$ In chicken, IGF2 is significantly associated with broilers growth traits and carcass traits, such as birth weight, breast muscle weight, abdominal fat weight and glandular stomach weight. ${ }^{18-20}$

Zinc finger E-box binding homeobox 1 (ZEB1) is a crucial nuclear transcription factor, it can directly bind to the E2-box [5-CATTC $(G)$ ] sequence in different gene promoter region, repressing the activity of gene transcription. ${ }^{21}$ In mammal, ZEB1 has three inhibiting zones. ${ }^{22}$ Zone-I mainly regulates T-lymphocyte differentiation. No inhibitory activity was found in zone-II. Zone-III is associated with muscle differentiation. In chicken, ZEB1 is also called DeltaEF1 (delta-crystallin/E2-box factor 1), which is highly homologous with mice and can also recognize the E2-box sequence (CATTCG). ${ }^{23}$ In the process

\footnotetext{
${ }^{1}$ Department of Animal Genetics, Breeding and Reproduction, College of Animal Science, South China Agricultural University, Guangzhou 510642, Guangdong Province, China; ${ }^{2}$ Guangdong Provincial Key Lab of Agro-Animal Genomics and Molecular Breeding, and Key Lab of Chicken Genetics, Breeding and Reproduction, Ministry of Agriculture, South China Agricultural University, Guangzhou 510642, Guangdong Province, China and ${ }^{3}$ Department of Cell Biology, School of Basic Medical Sciences, Southern Medical University, Guangzhou 510515, China

*Corresponding author: Q Nie, Department of Animal Genetics, College of Animal Science, South China Agricultural University, Guangzhou 510642, Guangdong Province, China. Tel: +86 20 85285759; Fax: +86 20 85280740; E-mail: nqinghua@scau.edu.cn

${ }^{4}$ These authors contributed equally to this work.

Received 31.5.17; revised 23.8.17; accepted 24.8.17; Edited by A Stephanou
} 
a

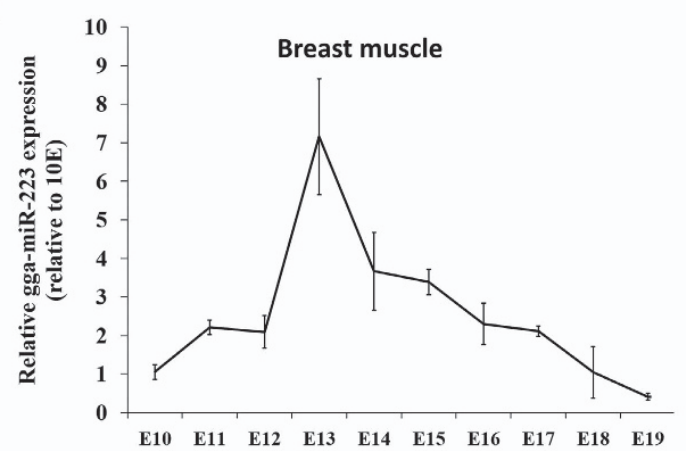

b

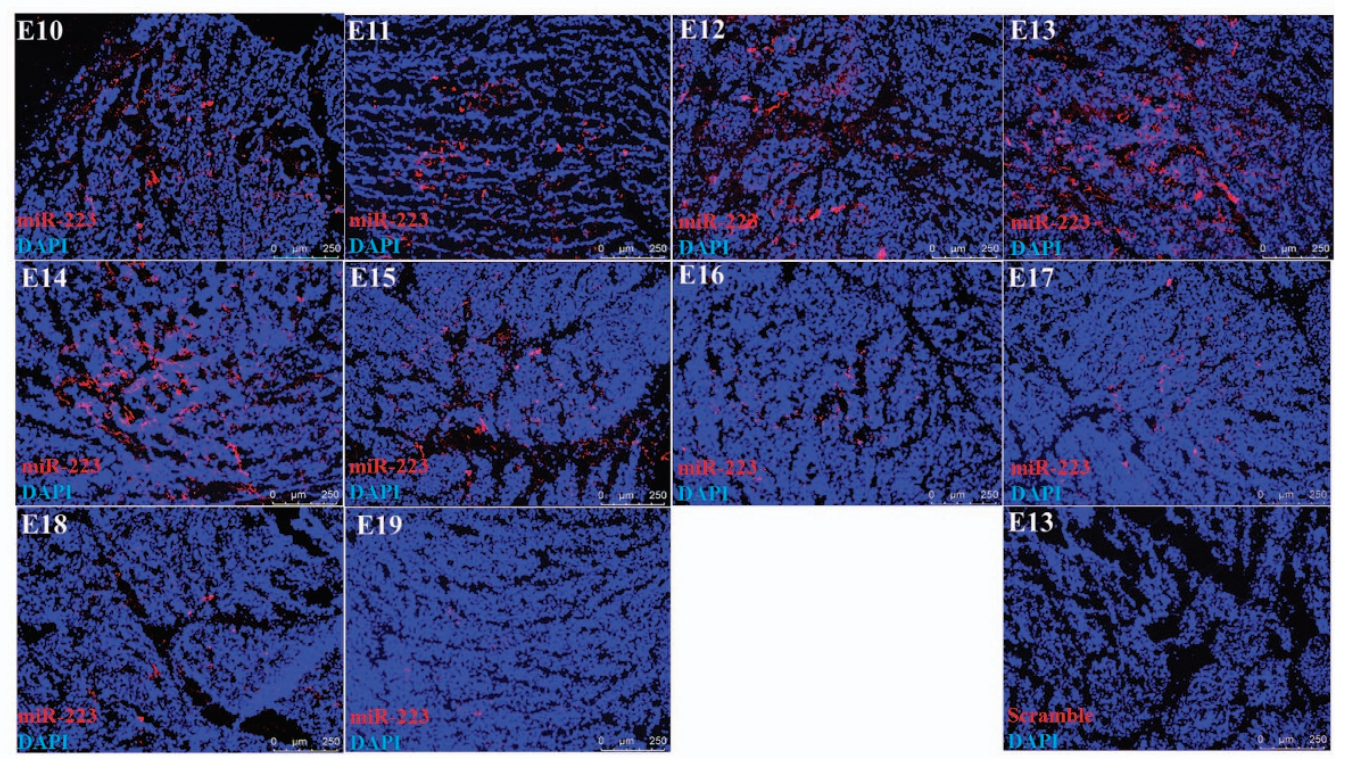

c

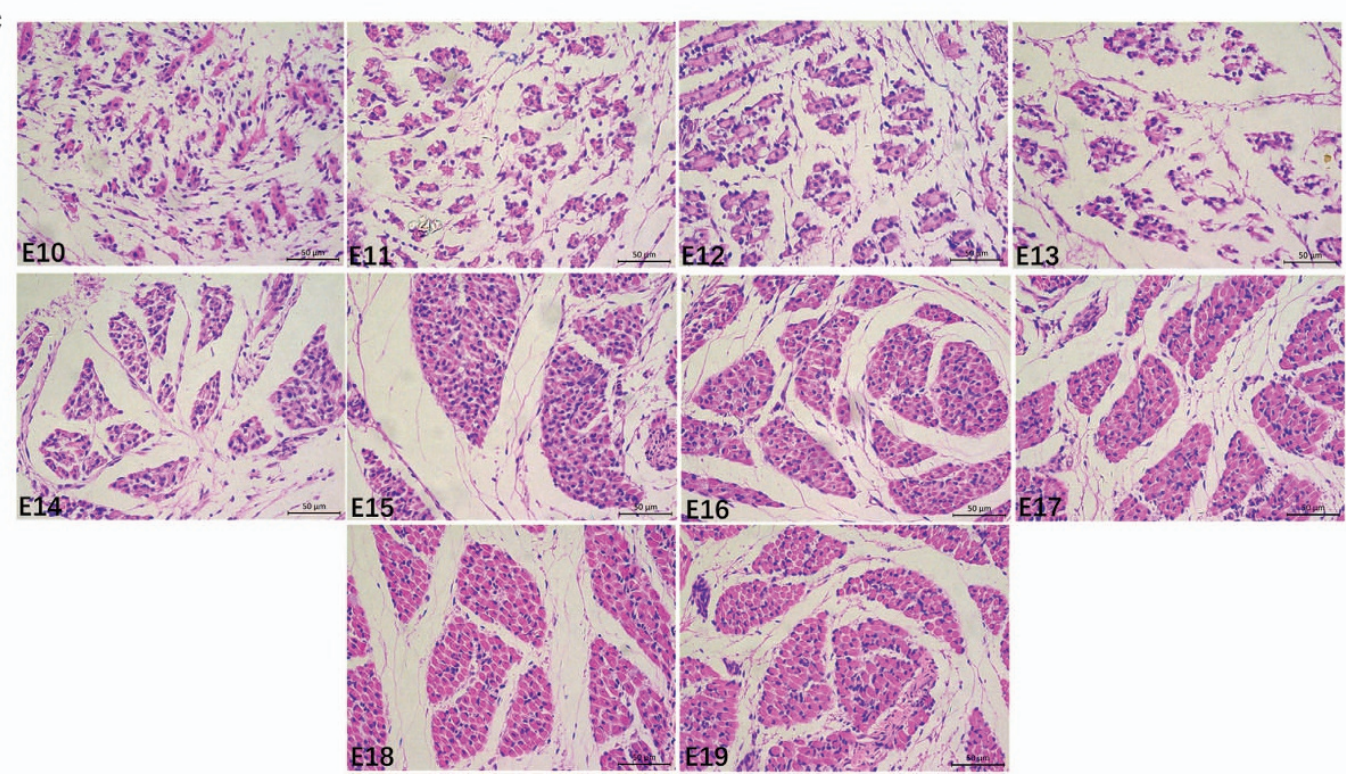

Figure 1 miR-223 expression is related to skeletal muscle development. (a) Relative expression of miR-223 in Xinghua chicken breast muscle from embryo day 10 (E10) to E19. (b) Fluorescence in situ hybridization of miR-223 in Xinghua chicken breast muscle from E10 to E19. (c) H-E staining of breast muscle fiber cross section from E10 to E19 in Xinghua chicken 

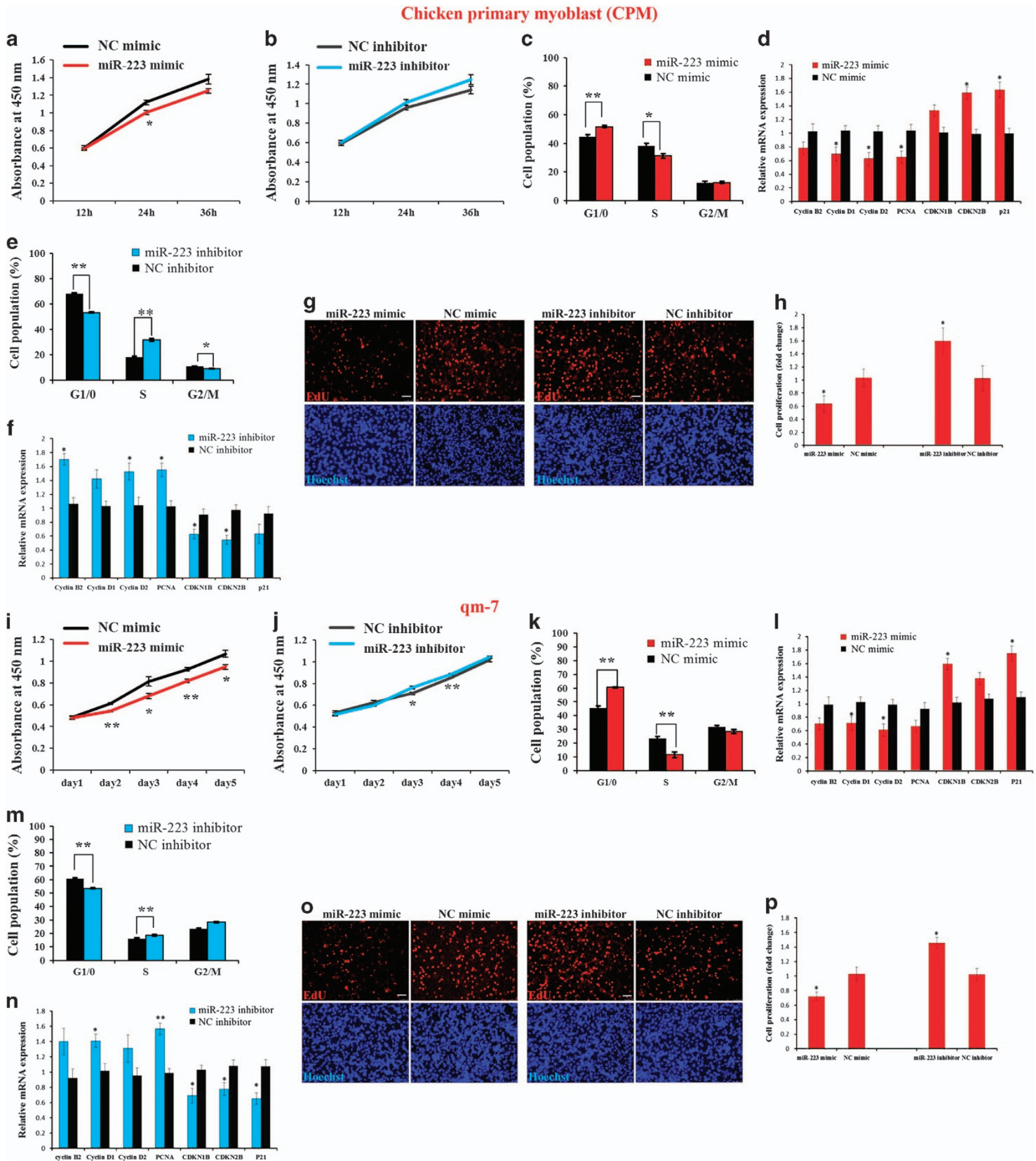

of chicken embryo development, ZEB1 was expressed in somite, notochord, urogenital system, nervous system and other parts of the chicken embryo, suggesting that it may involve in cell development process. ${ }^{24}$ In addition, ZEB1 can inhibit the activity of viral gene transcription by targeting the E2-box sequence exists in the promoter region of chicken infectious anemia virus, result in the decrease of the virus transcription activity. ${ }^{25}$
MYOD and MYOG are critical transcription factors in skeletal muscle differentiation and can regulate the transcription of most myogenesis-related genes. ${ }^{26-28}$ During myoblast differentiation, both of these two factors are upregulated their expression. ${ }^{29-31}$ MYOD and MYOG regulates their target genes transcription by binding to E-box promoter elements containing the site CANNTG. ${ }^{28}$ Recently, many myogenic miRNAs were found to be regulated by MYOD and MYOG, ${ }^{32}$ 
Figure 2 miR-223 inhibits myoblast proliferation. (a) CCK-8 assay indicates miR-223 overexpression inhibited chicken primary myoblast (CPM) proliferation. (b) CCK-8 assay indicates miR-223 inhibition promoted CPM proliferation. (c) CPM was overexpressed with miR-223 and the negative control (NC) mimic, and the cell cycle phase was then analyzed. (d) Relative mRNA expression of the cell cycle-related genes after transfection of miR-223 and NC mimic. (e) CPM was transfected with miR-223 inhibitor and the NC inhibitor, and the cell cycle phase was then analyzed. (f) Relative mRNA expression of the cell cycle-related genes after transfection of miR-223 inhibitor and NC inhibitor. (g) EdU staining after transfection of miR-223 mimic and miR-223 inhibitor. Bar, $50 \mu \mathrm{m}$. (h) The proliferation rate of myoblasts transfected with miR-223 mimic and miR-223 inhibitor. (i) CCK-8 assay showed that miR-223 overexpression inhibited qm-7 proliferation. (j) CCK-8 assay showed that miR-223 inhibition promoted qm-7 proliferation. (k) qm-7 was overexpressed with miR-223 and the NC mimic, and the cell cycle phase was then analyzed. (I) Relative mRNA expression of the cell cycle-related genes after transfection of miR-223 and NC mimic. (m) qm-7 was transfected with miR-223 inhibitor and the NC inhibitor, and the cell cycle phase was then analyzed. (n) Relative mRNA expression of the cell cycle-related genes after transfection of miR-223 inhibitor and NC inhibitor. (o) EdU staining after transfection of miR-223 mimic and miR-223 inhibitor. Bar, $50 \mu \mathrm{m}$. (p) The proliferation rate of qm-7 cells transfected with miR-223 mimic and miR-223 inhibitor. Results are shown as the mean \pm S.E.M. of three independent experiments. Independent sample $t$-test was used to analysis the statistical differences between groups. ${ }^{*} P<0.05 ;{ }^{*} P<0.01$

demonstrating an indirect role of these transcription factors in regulating muscle differentiation.

In this study, we investigated the function and regulation of miR-223 in avian skeletal muscle development. We found that miR-223 is regulated by MYOD transcription factor, which can bind to the promoter region of the gga-miR-223 gene. IGF2 and ZEB1 are two target genes of miR-223, and the upregulation of miR-223 can inhibit myoblast proliferation and promote myoblast differentiation by its repression on these two target genes. In addition, miR-223 represses IGF2 expression only in proliferating myoblast, and it has a more significant inhibiting effect on ZEB1 in differentiating myoblast than in proliferating myoblast. These results identified a role for miR-223 in avian myoblast proliferation and differentiation through dynamic regulates its target genes expression, and found another regulatory pathway of MYOD to shape myoblast differentiation.

\section{Results}

miR-223 expression is related to skeletal muscle development. In our previous miRNA sequencing data, we found that miR-223 exhibited differentially expression between the skeletal muscles of chickens with different growth rate. ${ }^{8}$ To further understand the relationship between miR-223 and skeletal muscle development, we detected its expression in breast muscle during chicken embryonic development. miR-223 was gradually upregulated its expression from embryo day 10 (E10) to E13. However, its expression was continually downregulated after E13 (Figure 1a). In addition, in situ hybridization results also showed that the miR-223 expression was upregulated from E10 to E13 and downregulated from E14 to E19 (Figure 1b). Haematoxylin-eosin staining of the breast muscle showed that the muscle fibers are blurry, irregular and may in the stage of cell differentiation and fusion between E10 and E13 (Figure 1c). After E13, the cross sections of muscle fibers were relatively regular and becoming more clearly defined (Figure 1c). Together with the expression data of miR-223, these results suggesting that miR-223 upregulated its expression during the active stage of muscle cell differentiation and fusion, whereas it downregulated its expression during muscle fiber maturation.

miR-223 inhibits myoblast proliferation. To further determine the roles of miR-223 in chicken skeletal muscle development, we transfected miR-223 mimics and inhibitors into chicken primary myoblast (CPM), respectively. CCK-8 assay indicated that miR-223 overexpression inhibited myoblast proliferation (Figure 2a), whereas miR-223 loss-offunction promoted myoblast proliferation (Figure 2b). In addition, by analyzing cell cycle of the transfected myoblasts, we found that miR-223 overexpression can decrease cell population in the $S$ phase and increase cell population in the G1/0 phase (Figure 2c). miR-223 overexpression can also inhibit the expression of cell cycle-promoting genes and enhance cell cycle-inhibiting genes (Figure 2d). On the contrary, inhibition of miR-223 significantly increased cell population in the $S$ phase and decreased cell population in the $\mathrm{G} 1 / 0$ phase (Figure 2e). miR-223 inhibition also enhanced the expression of cell cycle-promoting genes and repressed cell cycle-inhibiting genes (Figure $2 \mathrm{f})$. EdU staining showed that the proliferation rate of miR-223-transfected cells was significantly reduced compared with that of the control cells (Figure $2 \mathrm{~g}$ and $\mathrm{h}$ ), whereas miR-223 loss-of-function promoted cell proliferation rate (Figure $2 \mathrm{~g}$ and $\mathrm{h}$ ). Furthermore, these roles of miR-223 were also existed in the qm-7 cells (Figure $2 \mathrm{i}-\mathrm{p}$ ), indicating that miR-223 can inhibit avian myoblast proliferation.

The inhibitory effect of miR-223 on myoblast proliferation was achieved by its target gene IGF2. IGF2 is an important growth factor that can functioning as growth promoting hormone during cell development. Here, we found that the 3'-untranslated regions (3'-UTR) of IGF2 mRNA has a potential binding site of miR-223 (Figure 3a). To validate whether IGF2 is a target gene of miR-223, we constructed two dual-luciferase reporters with the wide-type and mutant $3^{\prime}$-UTR of IGF2, respectively. Results shown that miR-223 significantly repressed the luciferase activity of the wide-type reporter, whereas it has no effect on the luciferase activity of the mutant reporter (Figure 3b). In addition, miR-223 significantly inhibited the mRNA and protein expression of IGF2 gene in CPM (Figure 3c), and the inhibition of miR-223 released IGF2 mRNA and protein expression (Figure 3d). The above results indicated that IGF2 is a direct target gene of miR-223.

Although IGF2 is a well-known growth promoting-gene, its roles in chicken myoblast proliferation have not been examined. In this study, we transfected pSDS-IGF2 and three IGF2 siRNAs in poultry myoblast to overexpressing or inhibiting IGF2 expression, respectively. pSDS-IGF2 transfection significantly increased IGF2 mRNA and protein expression, whereas the si-170, which is specifically designed for 
a

chicken Position 121-144 of IGF2 3'UTR $5^{\prime}$... GCCCCAACCAAACAACTGACACAA... NM 001030342.2

chicken/quail miR-223 3'- CCCCAUAAACUGUUUGACUGU - 5'

quail Position 121-144 of IGF2 3'UTR 5'...GCCCCAACCAAACAACTGACACAA... XM_015863842.1

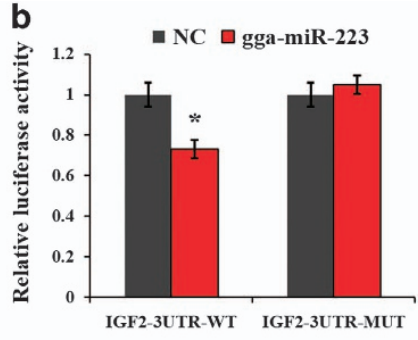

C

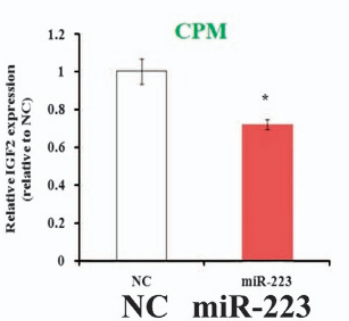

\begin{aligned} IGF2 & \multicolumn{2}{l}{$-\infty \mathrm{kDa}$} \\ GAPDH & $2 \mathrm{kDa}\end{aligned}$

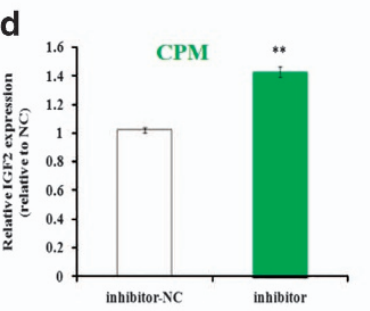

Inhibitor-NC Inhibitor-223
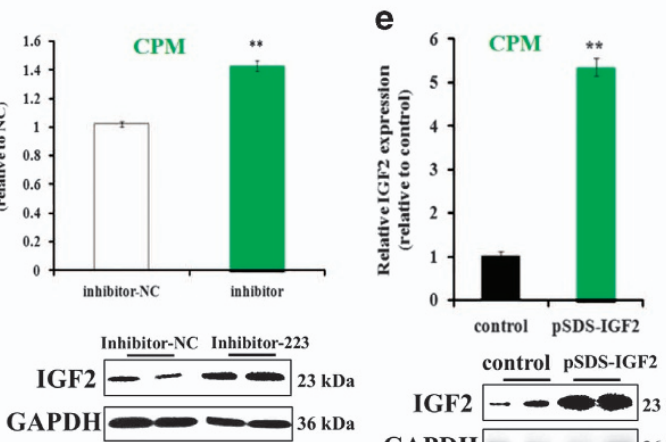

control pSDS-IGF2

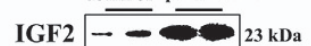

GAPDH $--\quad-36 \mathrm{kDa}$

f

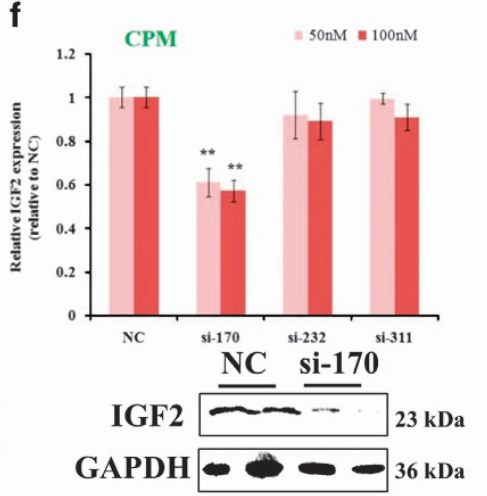

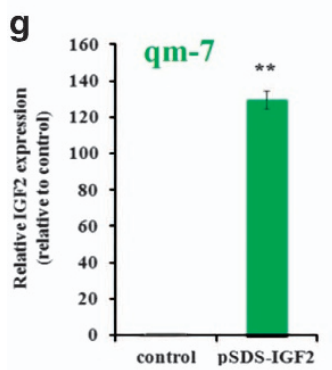
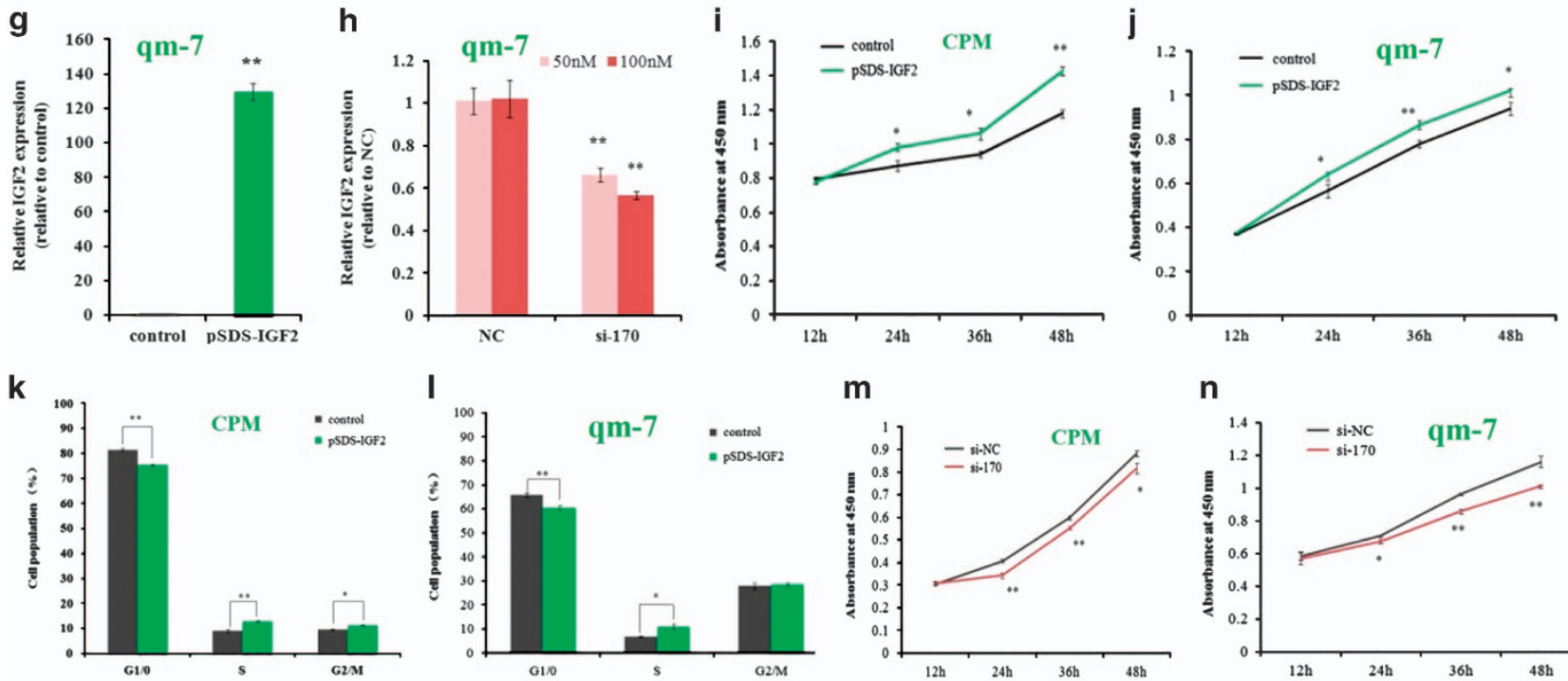

m

n
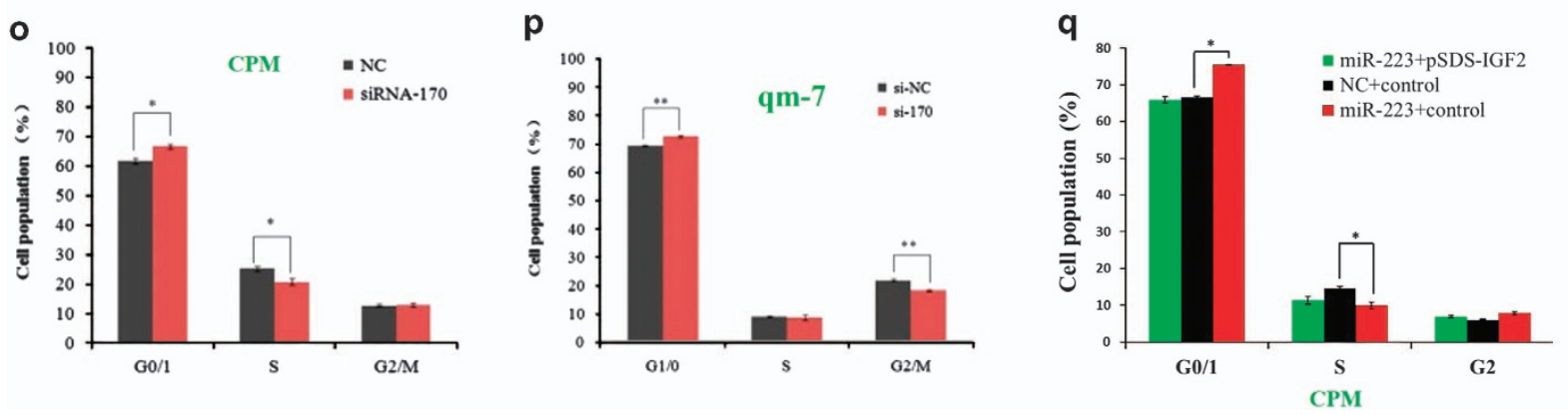

chicken IGF2 mRNA, significantly repressed IGF2 mRNA and protein expression (Figure 3e-h). IGF2 overexpression promotes myoblast proliferation through CCK-8 assay (Figure $3 \mathrm{i}$ and j) and cell cycle analysis (Figure $3 \mathrm{k}$ and I), whereas IGF2 inhibition repressed myoblast proliferation (Figure 3m-p). Furthermore, the rescue assay shown that miR-223 overexpression can inhibit the myoblast proliferation, and the transfection of IGF2 overexpression vector could counteract 
Figure 3 The inhibitory effect of miR-223 on myoblast proliferation was achieved by its target gene IGF2. (a) The potential binding site of miR-223 in the IGF2 mRNA 3' UTR. (b) Dual-luciferase reporter assay indicated that miR-223 can bind to the predicted binding site of the IGF2 mRNA 3' UTR. (c) miR-223 overexpression significantly inhibited IGF2 mRNA and protein expression in CPM. (d) miR-223 inhibition significantly promoted IGF2 mRNA and protein expression in CPM. (e) IGF2 overexpression significantly promoted IGF2 mRNA and protein expression in CPM. (f) IGF2 inhibition significantly inhibited IGF2 mRNA and protein expression in CPM. (g) IGF2 overexpression significantly promoted IGF2 mRNA and protein expression level in qm-7. (h) IGF2 inhibition significantly inhibited IGF2 mRNA and protein expression level in qm-7. (i) CCK-8 assay indicates that IGF2 overexpression significantly promoted myoblast proliferation in CPM. (j) CCK-8 assay indicates that IGF2 overexpression significantly promoted myoblast proliferation in qm-7. (k) CPM was overexpressed with IGF2 and the control vector, and the cell cycle phase was then analyzed. (I) qm-7 was overexpressed with IGF2 and the control vector, and the cell cycle phase was then analyzed. (m) CCK-8 assay indicates that IGF2 inhibition significantly reduced myoblast proliferation in CPM. (n) CCK-8 assay indicates that IGF2 inhibition significantly reduced myoblast proliferation in qm-7. (o) CPM was transfected with IGF2 siRNA and the NC siRNA, and the cell cycle phase was then analyzed. (p) qm-7 was transfected with IGF2 siRNA and the NC siRNA, and the cell cycle phase was then analyzed. (q). CPM was co-transfected with miR-223 and pSDS-IGF2, miRNA mimic NC and control vector, miR-223 and control vector, respectively, and the cell cycle phase was then analyzed. Results are shown as the mean \pm S.E.M. of three independent experiments. Independent sample $t$-test was used to analysis the statistical differences between groups. ${ }^{*} P<0.05 ;{ }^{*} P<0.01$

the inhibitory effect of miR-223 on myoblast proliferation (Figure $3 q$ ). Together, these results argue that the inhibitory effect of miR-223 on myoblast proliferation was achieved by its target gene IGF2.

miR-223 promotes myoblast differentiation. miR-223 was upregulated its expression during the active stage of chicken skeletal muscle differentiation and fusion. Next, we examined the expression and function of miR-223 during myoblast differentiation and fusion. miR-223 expression was significantly upregulated from the proliferation to the differentiation of both CPM and qm-7 cells (Figure $4 a$ and b), suggesting its involvement in these processes. Therefore, we transfected miR-223 mimic and inhibitor into the myoblasts. After transfection, the cells were induced to differentiation and fusion. Overexpression of miR-223 significantly increased the expression of muscle differentiation marker genes (Figure 4c and d), whereas the inhibition of miR-223 decreased the expression of these marker genes (Figure $4 \mathrm{e}$ and $\mathrm{f}$ ). In addition, miR-223 promotes the formation of myotubes (Figure $4 \mathrm{~g}$ ), and the size of the myotube area was significantly increased after transfection of miR-223 (Figure 4h). Therefore, these results demonstrated that miR-223 can promote myoblast differentiation.

ZEB1 is another miR-223 target gene, functioning as an inhibitor of myoblast differentiation. By using the TargetScan online software to predict the target genes of miR-223, we found that the $3^{\prime}$-UTR of ZEB1 mRNA has a potential binding site of miR-223 (Figure $5 a$ ). Notably, this binding site is conserved among vertebrates (Figure $5 b$ ). The dual-luciferase report assay suggested that miR-223 directly binds to the predicted target site of ZEB1-3'-UTR (Figure 5c). miR-223 overexpression inhibited the mRNA and protein expression level of ZEB1 (Figure $5 \mathrm{~d}$ and e), and the inhibition of miR-223 promoted the mRNA and protein expression level of ZEB1 (Figure $5 f$ and $g$ ). Therefore, the above results indicated that $Z E B 1$ is another target gene of miR-223.

During myoblast proliferation and differentiation, ZEB1 protein was gradually downregulated its expression (Figure $5 \mathrm{~h}$ ). To test the function of ZEB1 in myoblast differentiation, we synthesized siRNAs specifically target for chicken ZEB1 mRNA. Transfection of these siRNAs significantly inhibited the mRNA and protein expression level of ZEB1 in CPM and qm-7 (Figure 5i and j). In addition, ZEB1 inhibition significantly increased the expression of muscle differentiation marker genes in both of these two cells
(Figure 5k and I), suggesting ZEB1 is a repressor of myoblast differentiation. Rescue assay shown that ZEB1 overexpression is able to counteract the promotion effect of miR-223 on myoblast differentiation (Figure $5 \mathrm{~m}$ ). Therefore, ZEB1 is another miR-223 target gene that can function as an inhibitor of myoblast differentiation.

The inhibition of miR-223 on IGF2 and ZEB1 is different between myoblast proliferation and differentiation. The above results shown that both IGF2 and ZEB1 are target genes of miR-223. However, IGF2 is able to promote myoblast differentiation in mammal myoblast. ${ }^{33}$ Its expression was increased during CPM and qm-7 differentiation (Figure $6 a$ and b). This expression trend is similar to miR-223, which can directly inhibit IGF2 expression. In addition, specifically inhibition of IGF2 by siRNA significantly downregulated muscle differentiation marker genes (Figure $6 c$ and $d$ ), indicating its promoting effect in myoblast differentiation. These results suggested that miR-223 cannot restrict IGF2 expression and function during myoblast differentiation.

On the other hand, ZEB1, another miR-223 target gene that can inhibit myoblast differentiation, is unable to regulate cell cycle in proliferating myoblast (Figure 6e and f). Therefore, we were interest in how the miR-223 regulates the ZEB1 and IGF2 expression in the proliferating and differentiating myoblast, respectively. For ZEB1, miR-223 inhibits its expression in both proliferation and differentiation stages of myoblast (Figure $6 \mathrm{~g}$ ), and the inhibitory effect of miR-223 on ZEB1 has no significant difference between these two stages. For IGF2, miR-223 inhibits its expression only in the proliferation stage of myoblast (Figure $6 \mathrm{~g}$ ), and the miR-223 expression shown no significant difference between the proliferation and differentiation stages (Figure 6h). To further validate these results, we transfected dual-luciferase reporter inserted with the $3^{\prime}$-UTR of ZEB1 or IGF2 into the proliferating and differentiating myoblasts, respectively. The reporter activity inserted with ZEB1-3'-UTR was significant lower in differentiating myoblasts compared with that in proliferating myoblasts (Figure 6i). However, the reporter activity inserted with IGF2-3'-UTR was significant higher in differentiating myoblasts compared with that in proliferating myoblasts (Figure 6i). Together, these results suggested that the inhibition of miR-223 on IGF2 and ZEB1 is different between myoblast proliferation and differentiation.

MYOD regulates $m i R-223$ transcription by binding to the E-box 1 region. To further understand the structure of the 

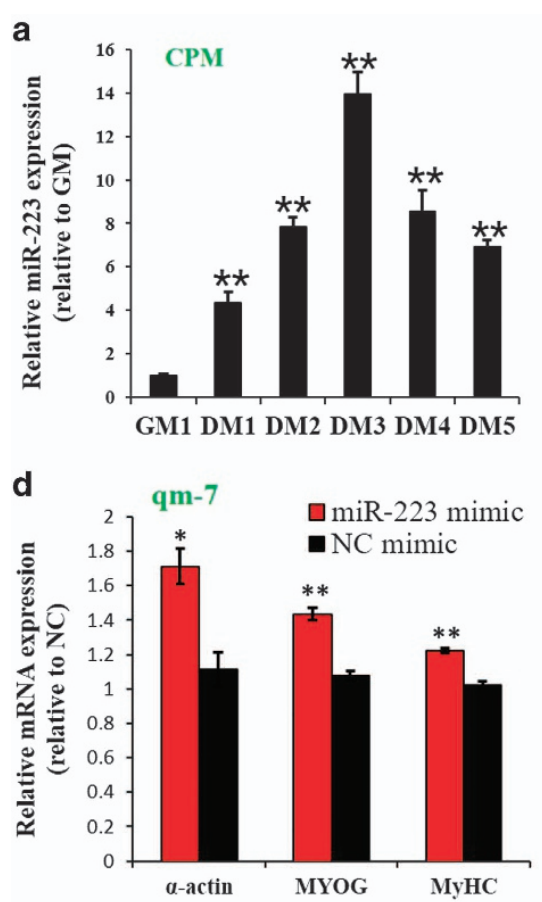

g
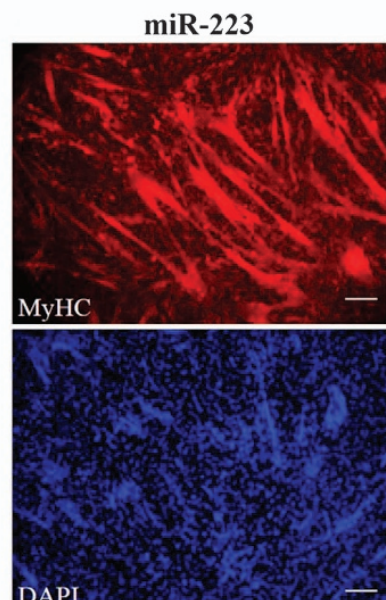

b

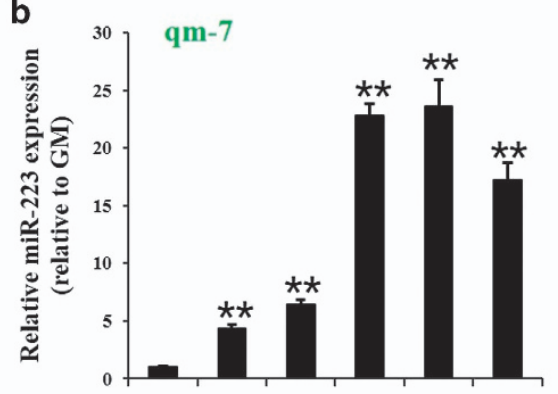

GM DM1 DM2 DM3 DM4 DM5

e

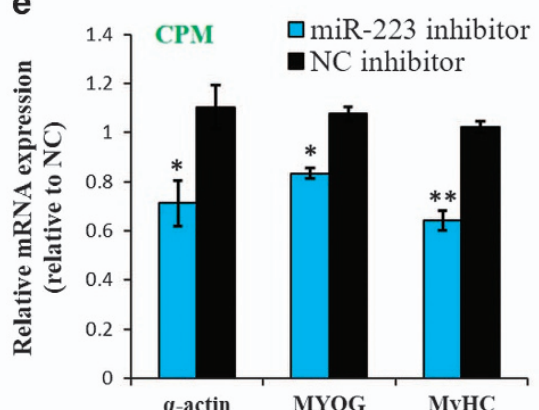

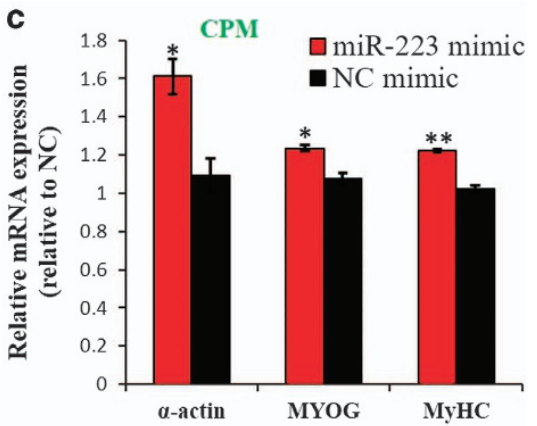

f

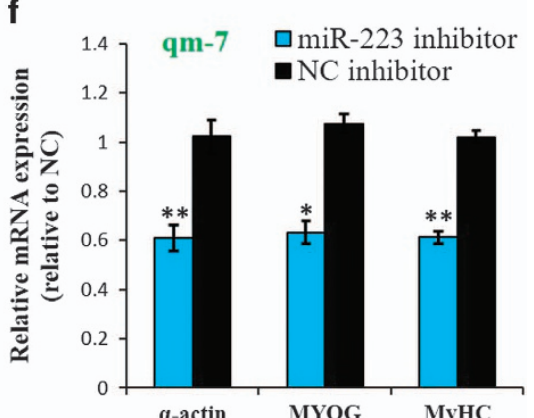

$\mathrm{NC}$

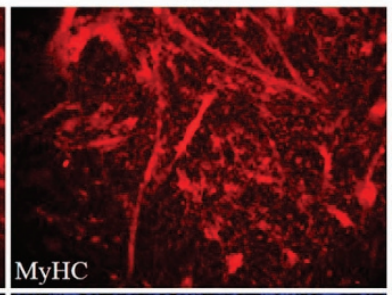

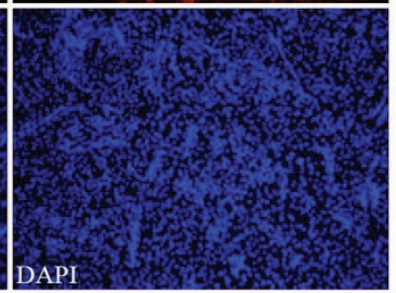

CPM

h

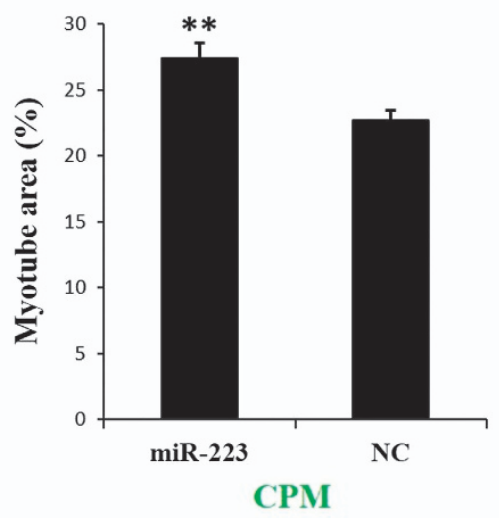

Figure 4 miR-223 promotes myoblast differentiation. (a) Relative miR-223 expression during CPM differentiation. (b) Relative miR-223 expression during qm-7 differentiation. (c) Relative expression of muscle differentiation marker genes after CPM transfected with miR-223 and NC. (d) Relative expression of muscle differentiation marker genes after qm-7 transfected with miR-223 and NC. (e) Relative expression of muscle differentiation marker genes after CPM transfected with miR-223 inhibitor and NC inhibitor. (f) Relative expression of muscle differentiation marker genes after qm-7 transfected with miR-223 inhibitor and NC inhibitor. (g) MyHC staining of CPM at $72 \mathrm{~h}$ after transfection of miR-223 or NC mimic. (h) Myotube area (\%) at $72 \mathrm{~h}$ after transfection of miR-223 or NC mimic. Results are shown as the mean \pm S.E.M. of three independent experiments. Independent sample $t$-test was used to analysis the statistical differences between groups. ${ }^{*} P<0.05 ;{ }^{*} P<0.01$

gga-miR-223 gene, we isolated its full-length pri-miR-223 by using $5^{\prime}$ and $3^{\prime}$ rapid amplification of cDNA ends (RACE). The obtained gga-miR-223 gene was $2228 \mathrm{bp}$ in length (Figure 7a). Next, we analyzed the upstream region of the gga-miR-223 gene to find the core promotor region. Four fragments, including 668-bp, 1020-bp, 1508-bp and 1932-bp upstream regions of the gga-miR-223 transcription start site (TSS) were amplified and cloned into the pGL3-basic vector. Forty-eight hours after transfected these reporter vectors into the myoblasts, the relative luciferase activity was measured to analyze the promoter activity of these four reporters. Results shown that the four reporters have similar relative luciferase activity (Figure $7 b$ ), suggesting that the shortest one or no reporter have the core promoter region. In addition, we found that there are two E-boxes located in the 1932-bp upstream regions of the gga-miR-223 TSS (Figure 7a). It is well-known that the MYOG and MYOD transcription factors can bind to the E-box region and promote the transcription activity of myogenic genes. ${ }^{34-36}$ Considering that MYOD, MYOG and miR-223 are all 
a

\begin{tabular}{cc}
\hline & $\begin{array}{c}\text { predicted consequential pairing of target region (top) and microRNA } \\
\text { (bottom) }\end{array}$ \\
\hline Position 62-68 of ZEB 1 3' UTR & 5' ...TATTAATACCCTTCTTACTGACATATG... \\
gea-miR-223 & | | | | | | | | । \\
\hline
\end{tabular}

b

Chicken CCUUCUUaCugacauauguaC Human CCUUCUUACUGACAUAUGUAC Chimp CCUUCUUACUGACAUAUGUAC

Rhesus CCUUCUUACUGACAUAUGUAC

Mouse CCUUCUUACUGACA - . CGUAC

Mouse CCUUCUUACUGACA - CGUAC

Rat CCUUCUUACUGACA - CGUAC

Rabbit CCUUCUUACUGACAUAUGUAC

Pig CCUUCUUACUGACAUAUGUAC

Cow CCUUCUUACUGACAUAUGUAC

Cat CCUUCUUACUGACAUGU - - AC

Dog CCUUCUUACUGACAUGU - - AC c

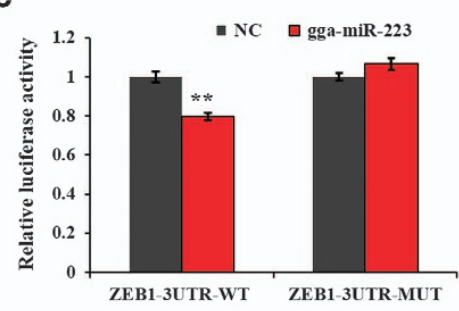

f

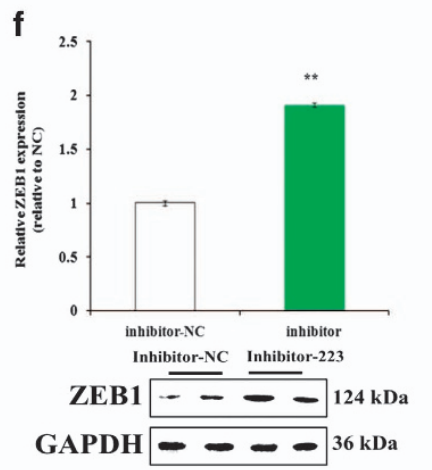

i
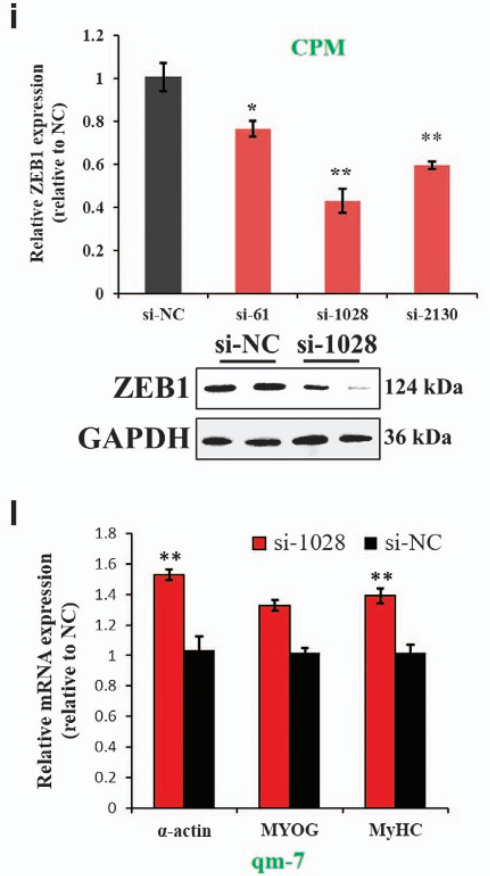

d

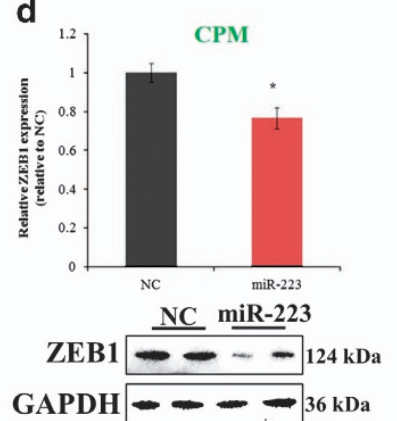

e

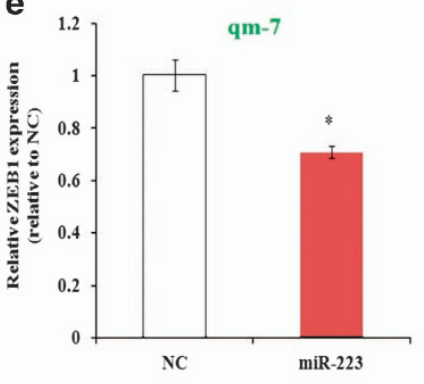

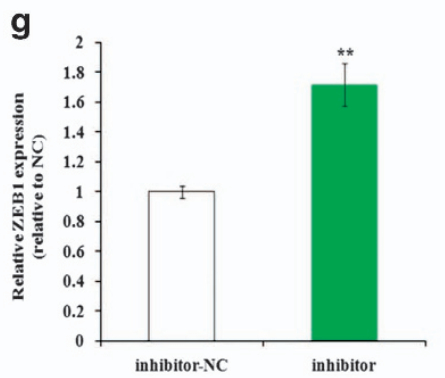

j

h
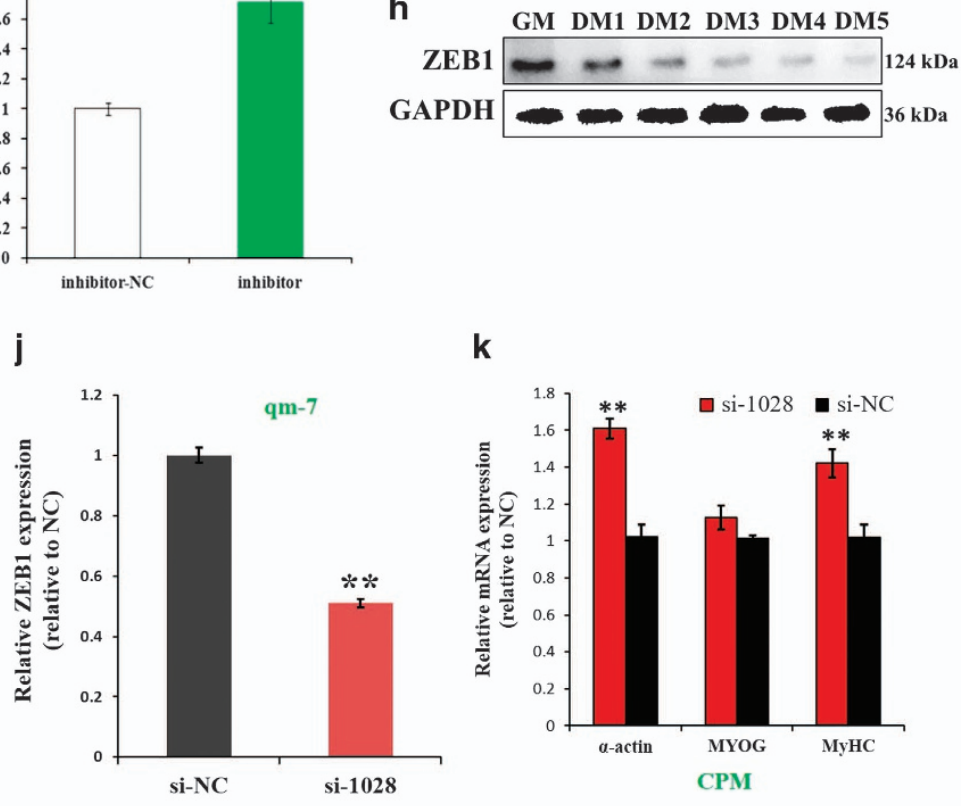

$\mathbf{k}$
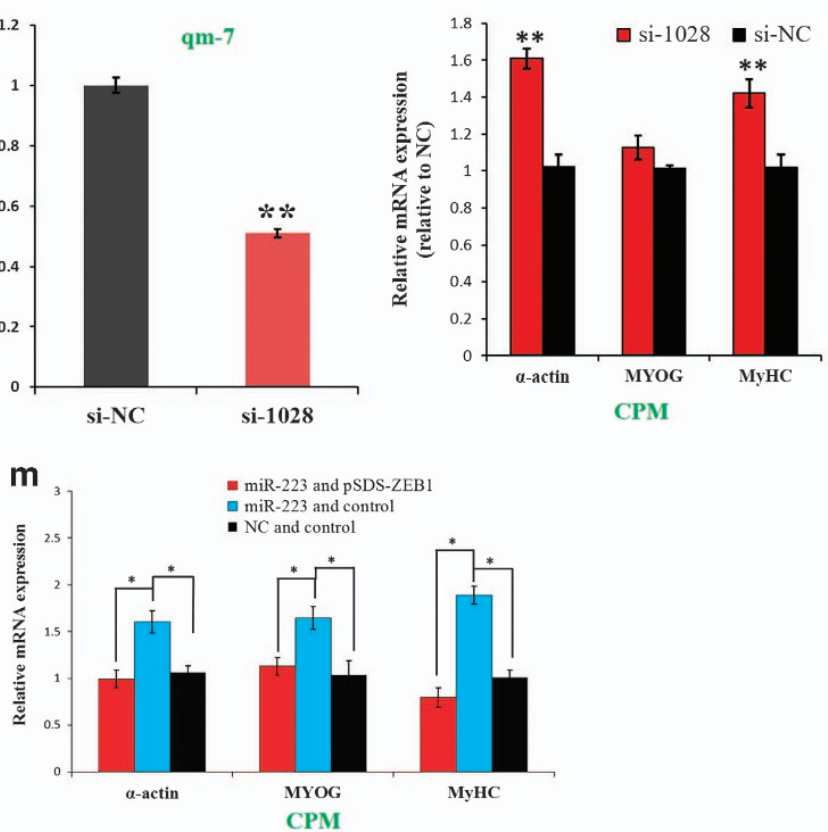

upregulated their expression during myoblast differentiation, we next examined whether MYOD and MYOG can bind to these two E-box regions and promote gga-miR-223 transcription. By mutated these two regions, respectively, we found that the relative luciferase activity was significantly reduced when we mutated E-box 1 , whereas there is no change of the luciferase activity when we mutated E-box 2 (Figure 7b). This result suggesting that the E-box 1 is 
Figure 5 ZEB1 is another miR-223 target gene, functioning as an inhibitor of myoblast differentiation. (a) The potential binding site of miR-223 in the ZEB1 mRNA 3' UTR. (b) The potential binding site (red) of miR-223 in the ZEB1 mRNA $3^{\prime}$ UTR is highly conserved among vertebrates. (c) Dual-luciferase reporter assay indicated that miR-223 can bind to the predicted binding site of the ZEB1 mRNA 3' UTR. (d) miR-223 overexpression inhibited ZEB1 mRNA and protein expression in CPM. (e) miR-223 overexpression inhibited ZEB1 mRNA expression in qm-7. (f) miR-223 inhibition promoted ZEB1 mRNA and protein expression in CPM. (g) miR-223 inhibition promoted ZEB1 mRNA expression in qm-7. (h) ZEB1 protein expression during CPM differentiation. (i) ZEB1 inhibition reduced ZEB1 mRNA and protein expression in CPM. (j) ZEB1 inhibition reduced ZEB1 mRNA expression in qm-7. (k) Expression level of muscle differentiation marker genes after CPM transfected with ZEB1 siRNA. (I) Expression level of muscle differentiation marker genes after qm-7 transfected with ZEB1 siRNA. (m) CPM was co-transfected with miR-223 and pSDS-IGF2, miRNA mimic NC and control vector, miR-223 and control vector, respectively, and expression level of muscle differentiation marker genes was then analyzed. Results are shown as the mean \pm S.E.M. of three independent experiments. Independent sample $t$-test was used to analysis the statistical differences between groups. ${ }^{*} P<0.05$; ${ }^{*} P<0.01$

a

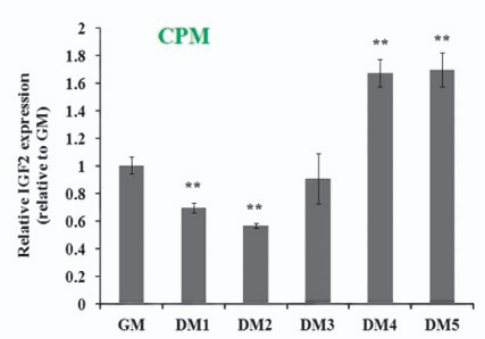

d

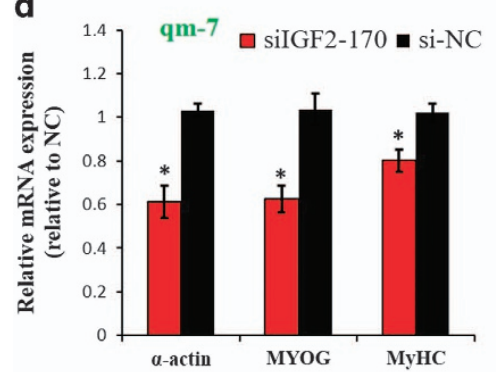

g

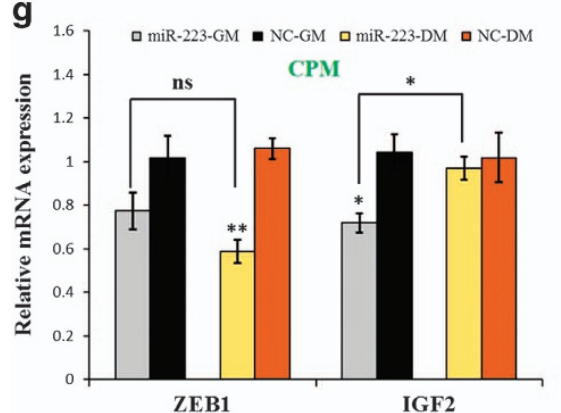

b

e
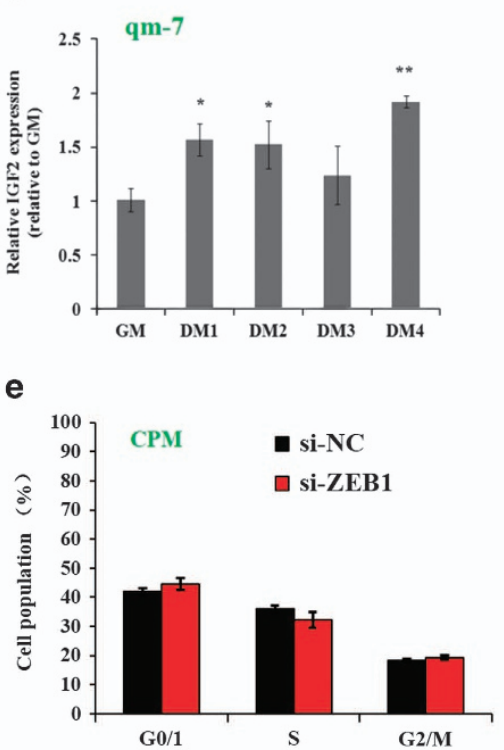

h

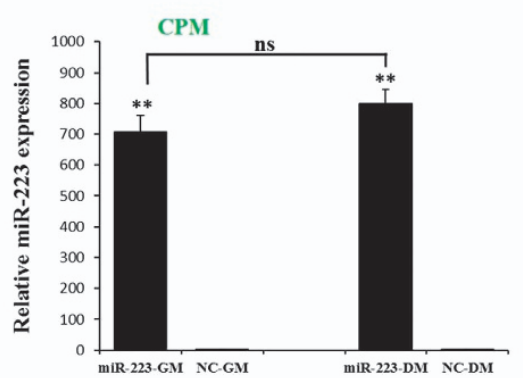

C

f
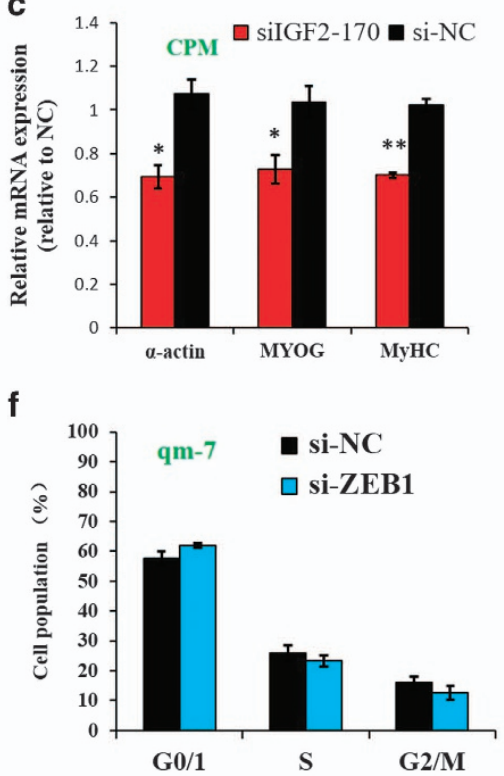

i

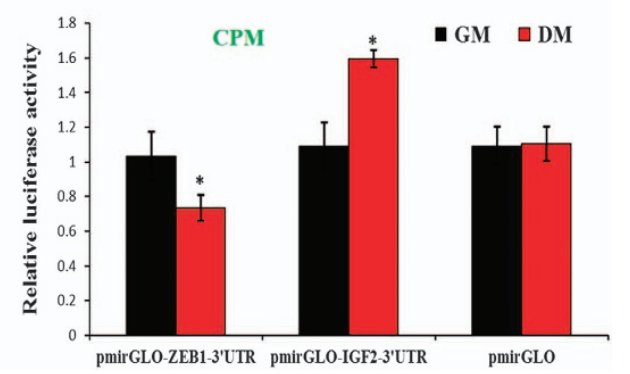

Figure 6 The inhibition of miR-223 on IGF2 and ZEB1 is different between myoblast proliferation and differentiation. (a) Relative IGF2 expression level during CPM differentiation. (b) Relative IGF2 expression level during qm-7 differentiation. (c) Expression level of muscle differentiation marker genes after CPM transfected with IGF2 siRNA. (d) Expression level of the muscle differentiation marker genes after qm-7 transfected with IGF2 siRNA. (e) CPM was transfected with ZEB1 siRNA and the NC siRNA, and the cell cycle phase was then analyzed. (f) qm-7 was transfected with ZEB1 siRNA and the NC siRNA, and the cell cycle phase was then analyzed. (g) The proliferating (GM) and differentiating (DM) CPM were transfected with miR-223 mimic, respectively, and the relative ZEB1 and IGF2 mRNA expression levels were then analyzed. (h) Relative miR-223 expression in proliferating and differentiating CPM after transfected with miR-223 and NC mimic. (i) The proliferating and differentiating CPM were transfected with pmirGLOZEB1-3'UTR, pmirGLO-IGF2-3'UTR or pmirGLO, and the relative luciferase activity was then analyzed. Results are shown as the mean \pm S.E.M. of three independent experiments. Independent sample $t$-test was used to analysis the statistical differences between groups. ns, no significant. ${ }^{*} P<0.05$; ${ }^{* \star} P<0.01$

important for the transcription activity of the promoter region. Next, chromatin immunoprecipitation assay (ChIP) results indicated that the MYOD can bind to the E-box 1 region (Figure 7c). To avoid the effect of endogenous MYOD, we used DF-1 cell to test the regulation of MYOD on the ggamiR-223 gene promoter and miR-223 transcription. MYOD overexpression promoted the relative luciferase activity of the pGL3-1932 reporter, but this overexpression had no effect on the luciferase activity of the mutated pGL3-1932 reporter (Figure 7d). In addition, MYOD overexpression promoted miR-223 expression (Figure 7e). For primary myoblast, MYOD loss-of-function not only inhibited the expression of miR-223 and muscle differentiation marker genes, but also promoted the expression of miR-223 target 
a

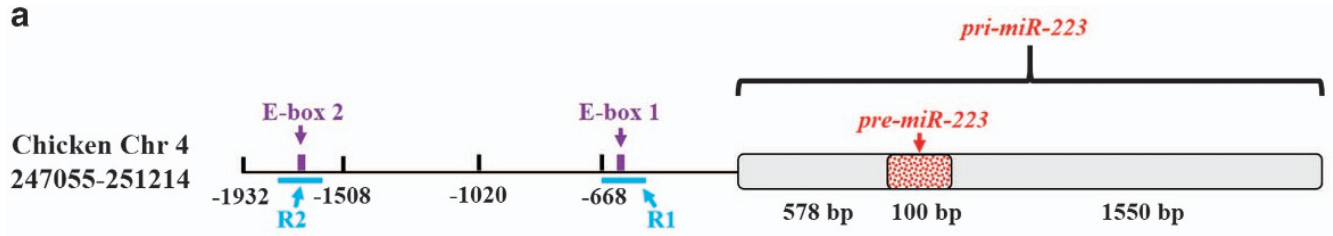

b

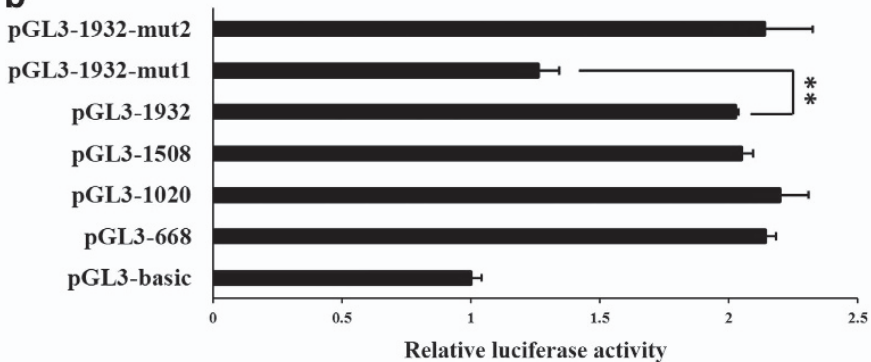

C

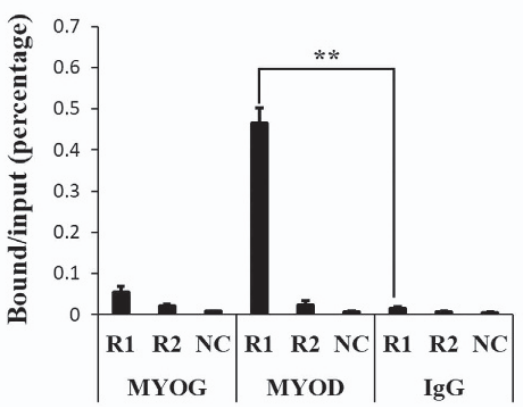

d
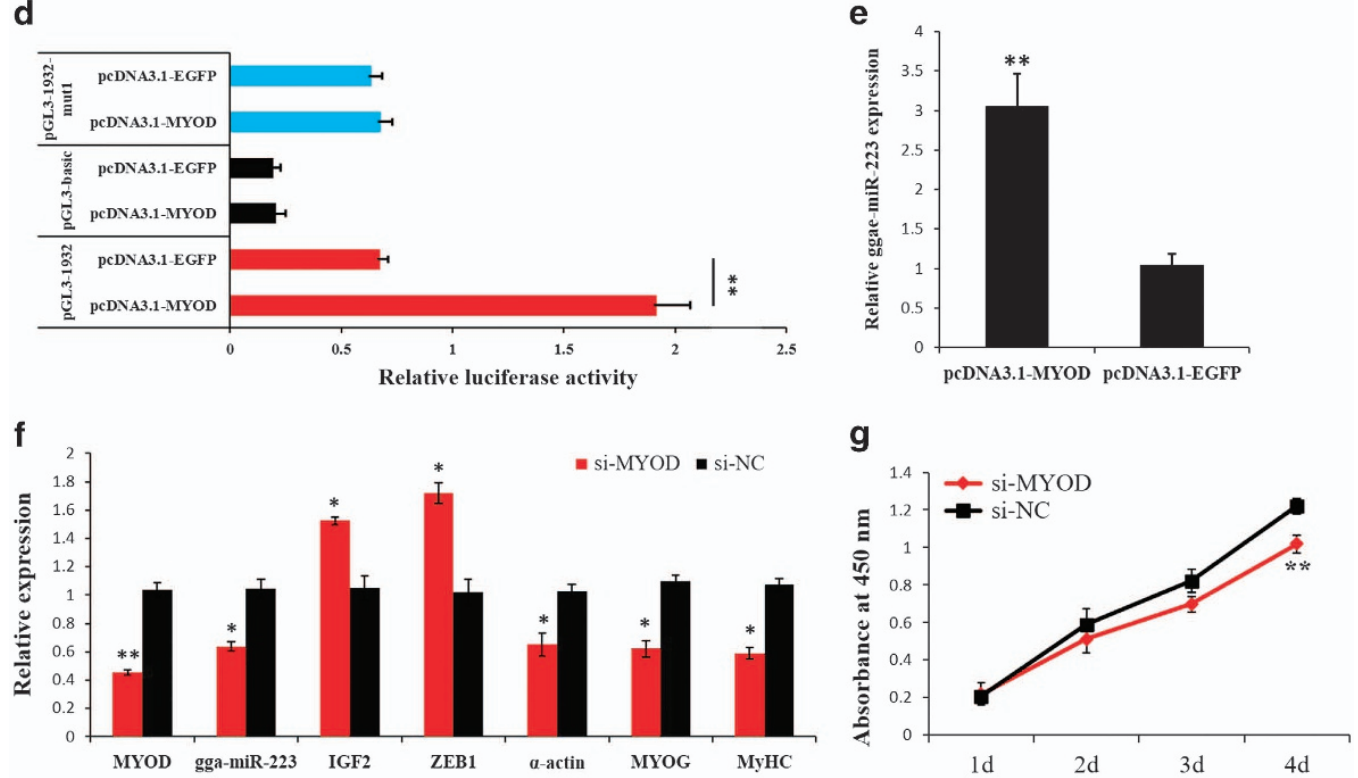

Figure 7 MYOD regulates miR-223 transcription by binding to the E-box 1 region. (a) The location of the two E-boxes in the 1932-kb upstream region of the gga-miR-223 gene TSS. (b) Identification of the core region in the gga-miR-223 gene promoter by luciferase reporter assays. (c) ChIP-qPCR analysis using anti-MYOG, anti-MYOD or chicken IgG antibodies, and the values showed that MYOD could bind to the R1 region of the chicken gga-miR-223 gene in myoblasts. A region from the GAPDH gene was amplified as a NC to verify the specificity of the enrichment. (d) MYOD overexpression promoted the relative luciferase activity of the pGL3-1932 reporter in DF-1 cell. (e) MYOD overexpression upregulated miR-223 expression in DF-1 cell. (f) Relative expressions of miR-223 and its downstream genes after transfection of si-MYOD in chicken primary myoblast. (g) CCK-8 assay indicated that MYOG loss-of-function significantly reduced myoblast proliferation in chicken primary myoblast. Results are shown as the mean \pm S.E.M. of three independent experiments. Independent sample $t$-test was used to analysis the statistical differences between groups. ${ }^{*} P<0.05 ;{ }^{*} P<0.01$ 
genes IGF2 and ZEB1 (Figure 7f). Furthermore, myoblast proliferation was also being inhibited when the cell was transfected with si-MYOD (Figure 7g). Together, these results demonstrated that the MYOD regulates ggamiR-223 transcription by binding to the E-box 1 region.

\section{Discussion}

In this study, we identified miR-223 as another miRNA that has a role in avian skeletal muscle development. miR-223 regulates myoblast proliferation and differentiation by balancing the expression of its target genes IGF2 and ZEB1. In addition, MYOD promotes miR-223 transcription during myoblast differentiation by binding to the gga-miR-223 gene promoter region (Figure 8). These findings not only revealed a new miRNA-mediated pathway functions on skeletal muscle differentiation, but also found an interest regulatory mode of miRNA in its target genes.

MYOD belongs to the myogenic basic-helix-loop-helix transcription factors, and is an important regulator during skeletal muscle differentiation. MYOD has the ability to initiate a lot of muscle-related genes transcription by recruitment of transcription factors and histone acetyltransferases. ${ }^{28,34,35}$ Many muscle development related genes have been found to be regulated by MYOD during myoblast differentiation. ${ }^{28,34}$ In this study, we found that MYOD can bind to the E-box, which is located in the promoter region of $m i R-223$ gene, and promote miR-223 expression in chicken myoblast. In addition, the expression of miR-223 and MYOD are upregulated during chicken myoblast differentiation, ${ }^{29}$ demonstrating a positive association between these two regulators. Besides, some of the muscle-specific miRNAs, such as miR-1, miR-133 and miR-206, can also be regulated by MYOD during myoblast differentiation. ${ }^{37,38}$ Therefore, these findings suggested that MYOD is able to regulate myoblast differentiation through activate various myogenic miRNAs expression.

ZEB1 is a transcriptional repressor. During muscle differentiation, ZEB1 is able to repressed muscle gene transcription, and the inhibition of ZEB1 can promote myoblast differentiation and fusion. ${ }^{39}$ Although the repression effect of ZEB1 in muscle differentiation has been well documented, however, its expression pattern during muscle differentiation still remains unknown. In this study, we have shown that ZEB1 protein was gradually downregulated during chicken myoblast differentiation, and the decrease of ZEB1 is, at least in part, owing to the inhibition of miR-223. In addition, ZEB1 can also repress myoblast differentiation in avian, but it cannot regulate myoblast proliferation, even though it has been shown to have positive role in many cancer cell proliferation. ${ }^{40-42}$ MYOD can occupy G/Ccentered E-boxes in the promoters of many musclerelated genes and initiate the transcription of these genes during myoblast differentiation. ${ }^{43}$ Interestingly, ZEB1 can also bind to the G/C-centered E-boxes in myoblast, but its binding results in the repression of muscle-related gene expression. ${ }^{39}$ Therefore, our results suggesting that MYOD inhibits ZEB1 expression by promoting miR-223 expression during myoblast differentiation.

IGF2 has long been established to have important roles in muscle development, ${ }^{44}$ and it is also a critical growth factor

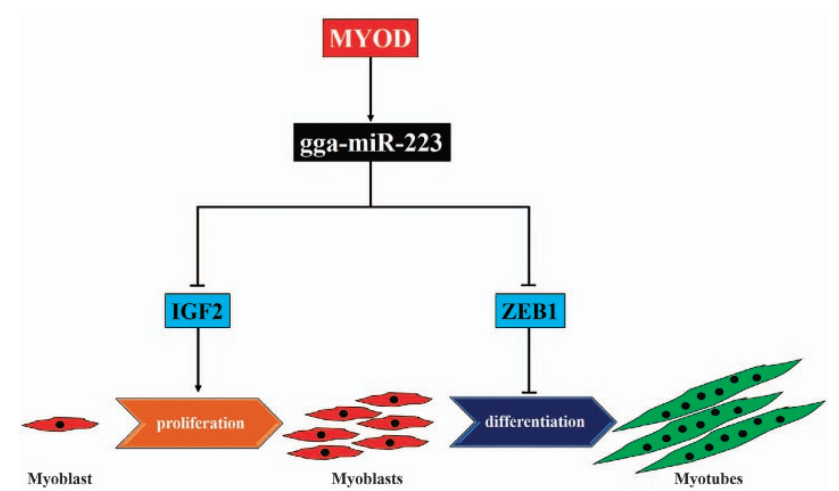

Figure 8 Model of the miR-223-mediated regulatory pathway for myoblast proliferation and differentiation

for chicken. ${ }^{19}$ However, the function of IGF2 in avian myoblast has not been verified before. Here, we reported that IGF2 can both promote avian myoblast proliferation and differentiation, and we also found that miR-223 has the ability to inhibit IGF2 expression. Notably, miR-223 inhibits IGF2 expression only in the proliferating myoblast. Its inhibition effect on IGF2 expression was significantly reduced from proliferating myoblast to differentiating myoblast. In addition, the inhibitory effect of miR-223 on ZEB1 in the proliferating myoblast was more significant than that in the differentiating myoblast. It is generally understood that a miRNA can bind to multiple target genes, and the inhibiting effect of miRNA on these target genes would be different because of their target sites with different functional properties. ${ }^{45}$ However, it is rare to see that a miRNA has different binding efficiency on one target gene during cell development. Previous study has shown that synaptic stimulation can relieve the repression of miRNA to its target genes. ${ }^{46,47}$ A miRNA-mediated inhibition of target gene translation can also be relieved when the cells subjected to different stress conditions, and this relief is depend on the binding of HuR, which is an AU-rich element RNA-binding protein (RBP), to the $3^{\prime}$ UTR of the target gene. ${ }^{48}$ Dead end 1 (Dnd1) is another RBP evolutionary conserved among vertebrates, and also has the ability to counteracts the inhibition roles of several miRNAs by binding to the $3^{\prime}$ UTR of target genes. ${ }^{49}$ However, the precise mechanism of how miR-223 dynamic regulates IGF2 and ZEB1 during myoblast proliferation and differentiation still need to be explored. Collectively, these and our findings suggest that the binding of miRNA to its target genes is dynamic in different cellular environment, and this binding can be rapidly responded to the specific cellular needs. ${ }^{48}$

In summary, this work has shown that the avian myoblast proliferation and differentiation are regulated by MYODmiR-223-IGF2/ZEB1 pathway. In the proliferating avian myoblast, miR-223, which is upregulated by MYOD, inhibits IGF2 expression to represses myoblast proliferation. In the differentiating avian myoblast, the upregulation of $M Y O D$ promotes miR-223 expression, and miR-223 inhibits ZEB1 rather than IGF2 to facilitate myoblast differentiation and fusion. 
Materials and Methods

Animals. The hatching eggs of Xinghua chickens were bought from the Chicken Breeding Farm of South China Agricultural University, Guangdong, China. More than ten chick embryos for each group were selected for pectorals and leg muscle separation. All the tissue samples were frozen in liquid nitrogen and stored at $-80^{\circ}$ $\mathrm{C}$ for the subsequent DNA and RNA extraction. With the sex-specific primers, the sex of each embryo was determined by PCR amplification.

RNA extraction, cDNA synthesis and quantitative real-time PCR. The total RNA was extracted from muscle tissues or cells using RNAiso reagent (Takara, Otsu, Japan). The reverse transcription reaction for mRNA was performed with PrimeScript RT reagent Kit (Perfect Real-Time) (Takara) according to manufacturer's manual. The reverse transcription reaction for miRNA was using ReverTra Ace qPCR RT Kit (Toyobo, Osaka, Japan). The specific Bulge-loop miRNA qRT-PCR Primer for miR-223 and U6 (one RT primer and a couple of qPCR primers for each gene) were designed by RiboBio (RiboBio, Guangzhou, China). With KAPA SYBR FAST qPCR Kit (KAPA Biosystems, Wobrun, MA, USA), qPCR program was carried out in Bio-rad CFX96 Real-Time Detection system (Bio-rad, Hercules, CA, USA), and the method was as described. ${ }^{50}$ All reactions were run in triplicate.

Cell culture. QM-7 cell culture: QM-7 cells were cultured in M199 (Gibco, Gaithersburg, MD, USA) supplemented with $10 \%$ fetal bovine serum (Hyclone, Logan, UT, USA), 10\% tryptose phosphate broth solution (Sigma Life Science, St. Louis, MO, USA) and $0.2 \%$ penicillin/streptomycin (Invitrogen, Carlsbad, CA, USA). The differentiation of QM-7 was induced by M199 supplemented with 10\% tryptose phosphate broth solution (Sigma) and $0.2 \%$ penicillin/streptomycin.

CPM isolation and culture: The leg muscle of E11 chickens were used to isolate $\mathrm{CPM}$. When the skin and bones removed, the leg muscles were rapidly chopped into small pieces in petri dish containing DMEM (Gibco) media supplemented with $20 \%$ fetal bovine serum (Hyclone) and $0.2 \%$ penicillin/streptomycin. The muscle suspending liquid was shaken by vortexing $1 \mathrm{~min}$ and filtered to obtain single cell. Repeat the vortexing and filtering steps for $4-6$ times to release enough cells. The cells were then collected by centrifugation at $1000 \times g$ for $5 \mathrm{~min}$ at room temperature. Decant medium and discard. Add DMEM supplemented with 20\% FBS and $0.2 \%$ penicillin/streptomycin to resuspend cells completely. Serial plating was needed to remove fibroblasts and enrich myoblasts. The differentiation of myoblasts was induced by DMEM supplemented with $0.2 \%$ penicillin/streptomycin.

Immunofluorescence. The immunofluorescence was performed in CPM cultured in 24-well plates. Cells were fixed in $4 \%$ paraformaldehyde for 20 mins at room temperature and then washed three times with PBS (5 min for every turn). Subsequently, cells were treated with $0.1 \%$ Triton X-100 for 20 minutes and were blocked with goat serum for $30 \mathrm{~min}$. Then, the cells were incubated with anti-MyHC (DSHB, lowa City, IA, USA) at $4{ }^{\circ} \mathrm{C}$ overnight. After washed with PBS, the cells were treated with FITC-conjugated anti-rabbit IgG antibody and incubated in dark for $1 \mathrm{~h}$. The cell nuclei were stained with DAPI (Beyotime, Jiangsu, China). The total myotube area was calculated and measured as previously described. ${ }^{50}$

The 5'- and 3'-RACE. The SMARTer RACE cDNA Amplification Kit (Takara) was used to perform both $5^{\prime}$ - and $3^{\prime}$-RACE according to the user manual. The $5^{\prime}$ and $3^{\prime}$ RACE-Ready first-strand CDNA was synthesized using total RNA extracted from chicken skeletal muscle. Two rounds of PCR reaction were preformed. First round was carried out with Universal Primer Mix (UPM) provided by the supplier inside the kit and miR-223 specific outer primer, and second round with Nested Universal Primer (NUP) also provided by the supplier inside the kit and miR-223 specific inner primer. Both 5'- and 3'-RACE PCR products were cloned and sequenced. All the primers used in RACE PCR are summarized in Supplementary file.

\section{Plasmid construction}

pmirGLO dual-luciferase reporters: The 3'UTR fragment of IGF2 (NCBI Reference Sequence: NM_001030342.1) and ZEB1 (NM_205131.1) containing the binding sites were amplified by PCR from chicken embryonic leg muscle cDNA and then cloned into pmirGLO vector. The mutant vectors were constructed by PCR mutagenesis. Five seed sequences were successful mutated from ACTGA to CTGAG for IGF2-3'UTR vector, and from CTGAC to ACTGT for ZEB1-3'UTR vector.
Gene overexpression vector. The IGF2 overexpression vector was constructed according to the user manual of Easy Ligation Kit (Sidansai, Shanghai, China). IGF2 coding sequence (NCBI Reference Sequence: NM_001030342.1) was amplified from chicken embryonic leg muscle CDNA by PCR. The PCR product was cloned into the pSDS-20117 vector (Sidansai). The successful IGF2 overexpression vector was confirmed by DNA sequencing. The MYOD overexpression vector was constructed as previously described. ${ }^{29}$

miR-223 promoter reporter plasmid: A 1932-bp fragment of the miR-223 promoter was isolated by PCR using the primers listed in Supplementary file. After the PCR product was digested with Kpnl and Smal restriction sites, the insertion was ligated into the pGL3-basic vector (Promega, Madison, WI, USA) to create the expression vector pGL3-1932. After pGL3-1932 was sequenced, this construct was used as a template, and pGL3-1508, pGL3-1020 or pGL3-668 was isolated by PCR. Site-directed mutagenesis of E-box 1 and E-box 2 was carried out by PCR amplification and Dpnl digestion to remove the parental DNA.

Transfections. Transfections were performed with Lipofectamine 3000 reagent (Invitrogen) according to the manufacturer's direction. Nucleic acids were diluted in OPTI-MEM Medium (Gibco). All experiments were carried out at least three times independently.

RNA oligonucleotides. The miR-223 mimics, negative control (NC), miR-223 inhibitors, miRNA inhibitor NC, siRNA against chicken IGF2, ZEB1 and MYOD were all purchased from GenePharma (GenePharma, Shanghai, China).

Dual-luciferase reporter assay. The miRNA target verification assay was performed in QM-7 cells. Wild-type or mutant IGF2-3' UTR dual-luciferase reporter $(200 \mathrm{ng})$ and miR-223 mimic or NC duplexes $(50 \mathrm{nM})$ were co-transfected into QM-7 cells using the Lipofectamine 3000 reagent (Invitrogen) in 48-well plates. For the promoter assays, qm-7 cells and DF-1 cells were co-transfected with reporter plasmid and MYOD overexpression vector or control vector, and the TK-Renilla reporter was also co-transfected to each sample as an internal control. After $48 \mathrm{~h}$ transfection, cells were washed by PBS twice and the activities of Firefly and Renilla luciferase were measured according to the manual of Luc-pair Duo-Luciferase Assay Kit 2.0 (GeneCopoeia, Rockville, MD, USA). All the data were acquired by averaging the results from four independent repeats.

Cell cycle analysis. After $36 \mathrm{~h}$ transfection, primary myoblast or QM-7 cells cultured in 12-well plates were fixed in $75 \%$ ethanol overnight at $-20^{\circ} \mathrm{C}$. With the Cell Cycle Analysis Kit (Thermo Fisher Scientific, Waltham, MA, USA), the cells were analyzed by a BD Accuri C6 flow cytometer (BD Biosciences, San Jose, CA, USA). All the data were acquired by averaging the results from three independent experiments.

CCK-8 assay. Primary myoblast or QM-7 cells were cultured in 96-well plates. A total of $10 \mu \mathrm{l}$ of Cell counting kit-8 reagent was added into each well and incubated for $1 \mathrm{~h}$. The assay was repeated at different time points of $12,24,36$, $48 \mathrm{~h}$ or every $24 \mathrm{~h}$ after transfection. The absorbance was measured at $450 \mathrm{~nm}$ by a Model 680 Microplate Reader (Bio-Rad). All the data were acquired by averaging the results from six independent experiments.

Western blot. Western blot was performed as previously described..$^{50}$ The following antibodies were used: anti-IGF2 (Santa Cruz Biotechnology, CA, USA), anti-ZEB1 (abcam, Cambridge, MA, USA) and anti-GAPDH (Bioworld, St Louis Park, MN, USA).

ChIP assays. ChIP assay was performed as previously described. ${ }^{29}$ Samples were done in triplicate. The primer sequences for ChIP-qPCR analysis are shown in Supplementary file.

miRNA in situ hybridization assay. gga-miR-223 miRCURY LNA probe and a scrambled probe were synthesized (Exiqon, Copenhagen, Denmark). $10 \mu \mathrm{m}$ thick cryosections from breast muscle tissues of Xinhua chicken were fixed in $4 \%$ PFA for $15 \mathrm{~min}$ at room temperature, and then treated for $10 \mathrm{~min}$ with Proteinase $\mathrm{K}$. miRNA in situ hybridization was performed using the FISH kit (Exonbio Lab, Guangzhou, China). DNA was counterstained with DAPI (1 mg/ml). Images of miRNA signals in slides were captured by a Leica DMi8 fluorescent microscope. 
EdU assay. EdU assay was performed as previously described with the following modifications. ${ }^{29}$ Twelve hours after transfection, primary myoblasts were exposed to $10 \mu \mathrm{M}$ 5-ethynyl-2'-deoxyuridine (EdU; RiboBio) for $24 \mathrm{~h}$ at $37^{\circ} \mathrm{C}$, and the QM-7 cells were exposed to $50 \mu \mathrm{M}$ EdU for $2 \mathrm{~h}$ at $37^{\circ} \mathrm{C}$. In addition, for primary myoblasts, $1 \times$ Apollo reaction cocktail (RiboBio) was added to the cells and incubated for $30 \mathrm{~min}$. Whereas, in QM-7 cells, $1 \times$ Apollo reaction cocktail was added to the cells and incubated for $20 \mathrm{~min}$. The EdU-stained cells were visualized under a Leica DMi8 fluorescent microscope. The proliferation rate was calculated by the number of EDU-stained cells normalized to the number of Hoechst 33342stained cells.

Statistical analysis. Each experiment was repeated three times, and all results are represented as the mean \pm S.E.M. Independent sample $t$-test was used to perform the statistical significant difference between groups.

Ethics standards. All experimental protocols were approved by the South China Agricultural University Institutional Animal Care and Use Committee (approval ID: SCAU\#0014). And the methods were carried out in accordance with the regulations and guidelines established by this committee.

\section{Conflict of Interest}

The authors declare no conflict of interest.

Acknowledgements. This work was supported by Natural Scientific Foundation of China (31472090), China High-Tech Programs (2013AA102501), the China Agriculture Research System (CARS-41-G03), and the Program for New Century Excellent Talents in University (NCET-13-0803), and the Foundation for High-level Talents in Higher Education of Guangdong, China.

\section{Publisher's Note}

Springer Nature remains neutral with regard to jurisdictional claims in published maps and institutional affiliations.

1. Bartel DP. MicroRNAs: genomics, biogenesis, mechanism, and function. Cell 2004; 116 281-297.

2. Rengaraj D, Lee BR, Lee SI, Seo HW, Han JY. Expression patterns and miRNA regulation of DNA methyltransferases in chicken primordial germ cells. PLOS ONE 2011; 6: e19524.

3. Gschwend AR, Weingartner LA, Moore RC, Ming R. The sex-specific region of sex chromosomes in animals and plants. Chromosome Res 2012; 20: 57-69.

4. Kang $L$, Cui $X$, Zhang $Y$, Yang $C$, Jiang $Y$. Identification of miRNAs associated with sexual maturity in chicken ovary by Illumina small RNA deep sequencing. BMC Genomics 2013; 14 : 352.

5. Huang HY, Liu RR, Zhao GP, Li QH, Zheng MQ, Zhang JJ et al. Integrated analysis of microRNA and mRNA expression profiles in abdominal adipose tissues in chickens. Sci Rep 2015; 5: 16132

6. Han B, Lian L, Li X, Zhao C, Qu L, Liu C et al. Chicken gga-miR-103-3p targets CCNE1 and TFDP2 and inhibits MDCC-MSB1 cell migration. G3 (Bethesda) 2016; 6: 1277-1285.

7. Li Y, Wang X, Yu J, Shao F, Zhang Y, Lu X et al. MiR-122 targets the vanin 1 gene to regulate its expression in chickens. Poult Sci 2016; 95: 1145-1150.

8. Ouyang $H, H e X, L i ~ G, X u H$, Jia X, Nie $Q$ et al. Deep sequencing analysis of miRNA expression in breast muscle of fast-growing and slow-growing broilers. Int J Mol Sci 2015; 16: $16242-16262$

9. Chen CZ, Li L, Lodish HF, Bartel DP. MicroRNAs modulate hematopoietic lineage differentiation. Science 2004; 303: 83-86.

10. Fazi F, Rosa A, Fatica A, Gelmetti V, De Marchis ML, Nervi C et al. A minicircuitry comprised of microRNA-223 and transcription factors $\mathrm{NFI}-\mathrm{A}$ and $\mathrm{C} / \mathrm{EBPalpha}$ regulates human granulopoiesis. Cell 2005; 123: 819-831.

11. Masaki S, Ontsuka R, Abe Y, Muta K, Umemura T. Expression patterns of microRNAs 155 and 451 during normal human erythropoiesis. Biochem Biophys Res Commun 2007; 364: 509-514.

12. Yuan JY, Wang F, Yu J, Yang GH, Liu XL, Zhang JW. MicroRNA-223 reversibly regulates erythroid and megakaryocytic differentiation of K562 cells. J Cell Mol Med 2009; 13: 4551-4559.

13. Yang W, Lan X, Li D, Li T, Lu S. MiR-223 targeting MAFB suppresses proliferation and migration of nasopharyngeal carcinoma cells. BMC Cancer 2015; 15: 461.

14. Rinderknecht $E$, Humbel RE. Primary structure of human insulin-like growth factor II. FEBS Lett 1978; 89: 283-286.

15. Vasilatos-Younken R, Scanes CG. Growth hormone and insulin-like growth factors in poultry growth: required, optimal, or ineffective? Poult Sci 1991; 70: 1764-1780.
16. Darling DC, Brickell PM. Nucleotide sequence and genomic structure of the chicken insulin-like growth factor-II (IGF-II) coding region. Gen Comp Endocrinol 1996; 102 283-287.

17. Gerrard DE, Okamura CS, Ranalletta MA, Grant AL. Developmental expression and location of IGF-I and IGF-II mRNA and protein in skeletal muscle. J Anim Sci 1998; 76: 1004-1011.

18. Amills M, Jimenez N, Villalba D, Tor M, Molina E, Cubilo D et al. Identification of three single nucleotide polymorphisms in the chicken insulin-like growth factor 1 and 2 genes and their associations with growth and feeding traits. Poult Sci 2003; 82: 1485-1493.

19. Tang S, Sun D, Ou J, Zhang Y, Xu G, Zhang Y. Evaluation of the IGFs (IGF1 and IGF2) genes as candidates for growth, body measurement, carcass, and reproduction traits in Beijing You and Silkie chickens. Anim Biotechnol 2010; 21: 104-113.

20. Gholami M, Erbe M, Garke C, Preisinger R, Weigend A, Weigend S et al. Population genomic analyses based on 1 million SNPs in commercial egg layers. PLOS ONE 2014; 9 : e94509.

21. van Grunsven LA, Taelman V, Michiels C, Opdecamp K, Huylebroeck D, Bellefroid EJ. deltaEF1 and SIP1 are differentially expressed and have overlapping activities during Xenopus embryogenesis. Dev Dyn 2006; 235: 1491-1500.

22. Postigo AA, Dean DC. Differential expression and function of members of the zfh-1 family of zinc finger/homeodomain repressors. Proc Natl Acad Sci USA 2000; 97: 6391-6396.

23. Sekido R, Murai K, Funahashi J, Kamachi Y, Fujisawa-Sehara A, Nabeshima Y et al. The delta-crystallin enhancer-binding protein delta EF1 is a repressor of E2-box-mediated gene activation. Mol Cell Biol 1994; 14: 5692-5700.

24. Funahashi J, Sekido R, Murai K, Kamachi Y, Kondoh H. Delta-crystallin enhancer binding protein delta EF1 is a zinc finger-homeodomain protein implicated in postgastrulation embryogenesis. Development 1993; 119: 433-446.

25. Miller MM, Jarosinski KW, Schat KA. Negative modulation of the chicken infectious anemia virus promoter by COUP-TF1 and an E box-like element at the transcription start site binding deltaEF1. J Gen Virol 2008; 89: 2998-3003.

26. Berkes CA, Tapscott SJ. MyoD and the transcriptional control of myogenesis. Semin Cell Dev Biol 2005; 16: 585-595.

27. Braun T, Gautel M. Transcriptional mechanisms regulating skeletal muscle differentiation, growth and homeostasis. Nat Rev Mol Cell Biol 2011; 12: 349-361.

28. Cao Y, Kumar RM, Penn BH, Berkes CA, Kooperberg C, Boyer LA et al. Global and genespecific analyses show distinct roles for Myod and Myog at a common set of promoters. EMBO J 2006; 25: 502-511.

29. Luo W, Li E, Nie Q, Zhang X. Myomaker, regulated by MYOD, MYOG and miR-140-3p promotes chicken myoblast fusion. Int J Mol Sci 2015; 16: 26186-26201.

30. Davis RL, Weintraub $H$, Lassar $A B$. Expression of a single transfected cDNA converts fibroblasts to myoblasts. Cell 1987; 51: 987-1000.

31. Hasty P, Bradley A, Morris JH, Edmondson DG, Venuti JM, Olson EN et al. Muscle deficiency and neonatal death in mice with a targeted mutation in the myogenin gene. Nature 1993; 364: 501-506.

32. Luo W, Nie Q, Zhang X. MicroRNAs involved in skeletal muscle differentiation. J Genet Genomics 2013; 40: 107-116.

33. Ge Y, Sun Y, Chen J. IGF-II is regulated by microRNA-125b in skeletal myogenesis. J Cell Biol 2011; 192: 69-81.

34. Blum R, Vethantham V, Bowman C, Rudnicki M, Dynlacht BD. Genome-wide identification of enhancers in skeletal muscle: the role of MyoD1. Genes Dev 2012; 26: 2763-2779.

35. Cao Y, Yao Z, Sarkar D, Lawrence M, Sanchez GJ, Parker MH et al. Genome-wide MyoD binding in skeletal muscle cells: a potential for broad cellular reprogramming. Dev Cell 2010; 18: $662-674$.

36. Aziz A, Liu QC, Dilworth FJ. Regulating a master regulator: establishing tissue-specific gene expression in skeletal muscle. Epigenetics 2010; 5: 691-695.

37. Rosenberg MI, Georges SA, Asawachaicharn A, Analau E, Tapscott SJ. MyoD inhibits Fst11 and Utrn expression by inducing transcription of miR-206. J Cell Biol 2006; 175: 77-85.

38. Koutsoulidou A, Mastroyiannopoulos NP, Furling D, Uney JB, Phylactou LA. Expression of miR-1, miR-133a, miR-133b and miR-206 increases during development of human skeletal muscle. BMC Dev Biol 2011; 11: 34.

39. Siles L, Sanchez-Tillo E, Lim JW, Darling DS, Kroll KL, Postigo A. ZEB1 imposes a temporary stage-dependent inhibition of muscle gene expression and differentiation via CtBP-mediated transcriptional repression. Mol Cell Biol 2013; 33: 1368-1382.

40. Hou LK, Yu Y, Xie YG, Wang J, Mao JF, Zhang B et al. miR-340 and ZEB1 negative feedback loop regulates TGF-beta- mediated breast cancer progression. Oncotarget 2016; 7 : 26016-26026.

41. Zhang Y, Liu G, Wu S, Jiang F, Xie J, Wang Y. Zinc finger E-box-binding homeobox 1: its clinical significance and functional role in human thyroid cancer. Onco Targets Ther 2016; 9 : 1303-1310.

42. Gu Y, Zhao Y, Zhou Y, Xie Y, Ju P, Long $Y$ et al. Zeb1 is potwntial regulator of Six2 in the proliferation, apoptosis and migration of metanephric mesenchyme cells. Int J Mol Sci 2016; 17: pii: E1283.

43. Soleimani VD, Yin $H$, Jahani-Asl A, Ming $H$, Kockx CE, van ljcken WF et al. Snail regulates MyoD binding-site occupancy to direct enhancer switching and differentiation-specific transcription in myogenesis. Mol Cell 2012; 47: 457-468.

44. Florini JR, Magri KA, Ewton DZ, James PL, Grindstaff K, Rotwein PS. "Spontaneous" differentiation of skeletal myoblasts is dependent upon autocrine secretion of insulin-like growth factor-II. J Biol Chem 1991; 266: 15917-15923. 
45. Brennecke J, Stark A, Russell RB, Cohen SM. Principles of microRNA-target recognition. PLOS Biol 2005; 3: e85.

46. Ashraf SI, McLoon AL, Sclarsic SM, Kunes S. Synaptic protein synthesis associated with memory is regulated by the RISC pathway in Drosophila. Cell 2006; 124: 191-205.

47. Schratt GM, Tuebing F, Nigh EA, Kane CG, Sabatini ME, Kiebler M et al. A brain-specific microRNA regulates dendritic spine development. Nature 2006; 439: 283-289.

48. Bhattacharyya SN, Habermacher R, Martine U, Closs El, Filipowicz W. Relief of microRNAmediated translational repression in human cells subjected to stress. Cell 2006; 125 1111-1124.

49. Kedde M, Strasser MJ, Boldajipour B, Oude VJ, Slanchev K, le Sage C et al. RNA-binding protein Dnd1 inhibits microRNA access to target mRNA. Cell 2007; 131 1273-1286.

50. Luo W, Li G, Yi Z, Nie Q, Zhang X. E2F1-miR-20a-5p/20b-5p auto-regulatory feedback loop involved in myoblast proliferation and differentiation. Sci Rep 2016; 6: 27904. (c) (i) Cell Death and Disease is an open-access journal published by Nature Publishing Group. This work is licensed under a Creative Commons Attribution 4.0 International License. The images or other third party material in this article are included in the article's Creative Commons license, unless indicated otherwise in the credit line; if the material is not included under the Creative Commons license, users will need to obtain permission from the license holder to reproduce the material. To view a copy of this license, visit http://creativecommons.org/licenses/by/4.0/

(C) The Author(s) 2017

Supplementary Information accompanies this paper on Cell Death and Disease website (http://www.nature.com/cddis) 